University of Nebraska - Lincoln

DigitalCommons@University of Nebraska - Lincoln

Faculty Publications from the Department of Electrical \& Computer Engineering, Department Electrical and Computer Engineering

11-19-2004

\title{
THERMODYNAMIC MODELING, ENERGY EQUIPARTITION, AND NONCONSERVATION OF ENTROPY FOR DISCRETE-TIME DYNAMICAL SYSTEMS
}

\author{
Wassim H. Haddad \\ Georgia Institute of Technology, wm.haddad@aerospace.gatech.edu \\ Qing Hui \\ Georgia Institute of Technology, qing.hui@unl.edu \\ Sergey G. Nersesov \\ Georgia Institute of Technology, sergeynersesov@ae.gatech.edu \\ Vijaysekhar Chellaboina \\ The University of Tennessee, chellaboina@utk.edu
}

Follow this and additional works at: https://digitalcommons.unl.edu/electricalengineeringfacpub

Part of the Computer Engineering Commons, and the Electrical and Computer Engineering Commons

Haddad, Wassim H.; Hui, Qing; Nersesov, Sergey G.; and Chellaboina, Vijaysekhar, "THERMODYNAMIC MODELING, ENERGY EQUIPARTITION, AND NONCONSERVATION OF ENTROPY FOR DISCRETE-TIME DYNAMICAL SYSTEMS" (2004). Faculty Publications from the Department of Electrical and Computer Engineering. 521.

https://digitalcommons.unl.edu/electricalengineeringfacpub/521

This Article is brought to you for free and open access by the Electrical \& Computer Engineering, Department of at DigitalCommons@University of Nebraska - Lincoln. It has been accepted for inclusion in Faculty Publications from the Department of Electrical and Computer Engineering by an authorized administrator of DigitalCommons@University of Nebraska - Lincoln. 


\title{
THERMODYNAMIC MODELING, ENERGY EQUIPARTITION, AND NONCONSERVATION OF ENTROPY FOR DISCRETE-TIME DYNAMICAL SYSTEMS
}

\author{
WASSIM M. HADDAD, QING HUI, SERGEY G. NERSESOV, \\ AND VIJAYSEKHAR CHELLABOINA
}

Received 19 November 2004

We develop thermodynamic models for discrete-time large-scale dynamical systems. Specifically, using compartmental dynamical system theory, we develop energy flow models possessing energy conservation, energy equipartition, temperature equipartition, and entropy nonconservation principles for discrete-time, large-scale dynamical systems. Furthermore, we introduce a new and dual notion to entropy; namely, ectropy, as a measure of the tendency of a dynamical system to do useful work and grow more organized, and show that conservation of energy in an isolated thermodynamic system necessarily leads to nonconservation of ectropy and entropy. In addition, using the system ectropy as a Lyapunov function candidate, we show that our discrete-time, large-scale thermodynamic energy flow model has convergent trajectories to Lyapunov stable equilibria determined by the system initial subsystem energies.

\section{Introduction}

Thermodynamic principles have been repeatedly used in continuous-time dynamical system theory as well as in information theory for developing models that capture the exchange of nonnegative quantities (e.g., mass and energy) between coupled subsystems $[5,6,8,11,20,23,24]$. In particular, conservation laws (e.g., mass and energy) are used to capture the exchange of material between coupled macroscopic subsystems known as compartments. Each compartment is assumed to be kinetically homogeneous; that is, any material entering the compartment is instantaneously mixed with the material in the compartment. These models are known as compartmental models and are widespread in engineering systems as well as in biological and ecological sciences $[1,7,9,16,17,22]$. Even though the compartmental models developed in the literature are based on the first law of thermodynamics involving conservation of energy principles, they do not tell us whether any particular process can actually occur; that is, they do not address the second law of thermodynamics involving entropy notions in the energy flow between subsystems.

The goal of the present paper is directed towards developing nonlinear discrete-time compartmental models that are consistent with thermodynamic principles. Specifically, 
since thermodynamic models are concerned with energy flow among subsystems, we develop a nonlinear compartmental dynamical system model that is characterized by energy conservation laws capturing the exchange of energy between coupled macroscopic subsystems. Furthermore, using graph-theoretic notions, we state three thermodynamic axioms consistent with the zeroth and second laws of thermodynamics that ensure that our large-scale dynamical system model gives rise to a thermodynamically consistent energy flow model. Specifically, using a large-scale dynamical systems theory perspective, we show that our compartmental dynamical system model leads to a precise formulation of the equivalence between work energy and heat in a large-scale dynamical system.

Next, we give a deterministic definition of entropy for a large-scale dynamical system that is consistent with the classical thermodynamic definition of entropy and show that it satisfies a Clausius-type inequality leading to the law of entropy nonconservation. Furthermore, we introduce a new and dual notion to entropy; namely, ectropy, as a measure of the tendency of a large-scale dynamical system to do useful work and grow more organized, and show that conservation of energy in an isolated thermodynamically consistent system necessarily leads to nonconservation of ectropy and entropy. Then, using the system ectropy as a Lyapunov function candidate, we show that our thermodynamically consistent large-scale nonlinear dynamical system model possesses a continuum of equilibria and is semistable; that is, it has convergent subsystem energies to Lyapunov stable energy equilibria determined by the large-scale system initial subsystem energies. In addition, we show that the steady-state distribution of the large-scale system energies is uniform leading to system energy equipartitioning corresponding to a minimum ectropy and a maximum entropy equilibrium state. In the case where the subsystem energies are proportional to subsystem temperatures, we show that our dynamical system model leads to temperature equipartition, wherein all the system energy is transferred into heat at a uniform temperature. Furthermore, we show that our system-theoretic definition of entropy and the newly proposed notion of ectropy are consistent with Boltzmann's kinetic theory of gases involving an $n$-body theory of ideal gases divided by diathermal walls.

The contents of the paper are as follows. In Section 2, we establish notation, definitions, and review some basic results on nonnegative and compartmental dynamical systems. In Section 3, we use a large-scale dynamical systems perspective to develop a nonlinear compartmental dynamical system model characterized by energy conservation laws that is consistent with basic thermodynamic principles. Then we turn our attention to stability and convergence. In particular, using the total subsystem energies as a candidate system energy storage function, we show that our thermodynamic system is lossless and hence can deliver to its surroundings all of its stored subsystem energies and can store all of the work done to all of its subsystems. Next, using the system ectropy as a Lyapunov function candidate, we show that the proposed thermodynamic model is semistable with a uniform energy distribution corresponding to a minimum ectropy and a maximum entropy. In Section 4, we generalize the results of Section 3 to the case where the subsystem energies in large-scale dynamical system model are proportional to subsystem temperatures and arrive at temperature equipartition for the proposed thermodynamic model. 
Furthermore, we provide an interpretation of the steady-state expressions for entropy and ectropy that is consistent with kinetic theory. In Section 5, we specialize the results of Section 3 to thermodynamic models with linear energy exchange. Finally, we draw conclusions in Section 6.

\section{Mathematical preliminaries}

In this section, we introduce notation, several definitions, and some key results needed for developing the main results of this paper. Let $\mathbb{R}$ denote the set of real numbers, let $\overline{\mathbb{Z}}_{+}$ denote the set of nonnegative integers, let $\mathbb{R}^{n}$ denote the set of $n \times 1$ column vectors, let $\mathbb{R}^{m \times n}$ denote the set of $m \times n$ real matrices, let $(\cdot)^{\mathrm{T}}$ denote transpose, and let $I_{n}$ or $I$ denote the $n \times n$ identity matrix. For $v \in \mathbb{R}^{q}$, we write $v \geq \geq 0$ (resp., $v \gg 0$ ) to indicate that every component of $v$ is nonnegative (resp., positive). In this case, we say that $v$ is nonnegative or positive, respectively. Let $\overline{\mathbb{R}}_{+}^{q}$ and $\mathbb{R}_{+}^{q}$ denote the nonnegative and positive orthants of $\mathbb{R}^{q}$; that is, if $v \in \mathbb{R}^{q}$, then $v \in \overline{\mathbb{R}}_{+}^{q}$ and $v \in \mathbb{R}_{+}^{q}$ are equivalent, respectively, to $v \geq \geq 0$ and $v \gg 0$. Finally, we write $\|\cdot\|$ for the Euclidean vector norm, $\mathscr{R}(M)$ and $\mathcal{N}(M)$ for the range space and the null space of a matrix $M$, respectively, $\operatorname{spec}(M)$ for the spectrum of the square matrix $M, \operatorname{rank}(M)$ for the rank of the matrix $M, \operatorname{ind}(M)$ for the index of $M$; that is, $\min \left\{k \in \overline{\mathbb{Z}}_{+}: \operatorname{rank}\left(M^{k}\right)=\operatorname{rank}\left(M^{k+1}\right)\right\}, M^{\#}$ for the group generalized inverse of $M$, where $\operatorname{ind}(M) \leq 1, \Delta E(x(k))$ for $E(x(k+1))-E(x(k)), \mathscr{B}_{\varepsilon}(\alpha), \alpha \in \mathbb{R}^{n}, \varepsilon>0$, for the open ball centered at $\alpha$ with radius $\varepsilon$, and $M \geq 0$ (resp., $M>0$ ) to denote the fact that the Hermitian matrix $M$ is nonnegative (resp., positive) definite.

The following definition introduces the notion of $Z-, M-$, nonnegative, and compartmental matrices.

Definition $2.1[2,5,12]$. Let $W \in \mathbb{R}^{q \times q}$. $W$ is a $Z$-matrix if $W_{(i, j)} \leq 0, i, j=1, \ldots, q, i \neq j$. $W$ is an $M$-matrix (resp., a nonsingular $M$-matrix) if $W$ is a $Z$-matrix and all the principal minors of $W$ are nonnegative (resp., positive). $W$ is nonnegative (resp., positive) if $W_{(i, j)} \geq$ 0 (resp., $\left.W_{(i, j)}>0\right), i, j=1, \ldots, q$. Finally, $W$ is compartmental if $W$ is nonnegative and $\sum_{i=1}^{q} W_{(i, j)} \leq 1, j=1, \ldots, q$.

In this paper, it is important to distinguish between a square nonnegative (resp., positive) matrix and a nonnegative-definite (resp., positive-definite) matrix.

The following definition introduces the notion of nonnegative functions [12].

Definition 2.2. Let $w=\left[w_{1}, \ldots, w_{q}\right]^{\mathrm{T}}: \mathscr{V} \rightarrow \mathbb{R}^{q}$, where $\mathscr{V}$ is an open subset of $\mathbb{R}^{q}$ that contains $\overline{\mathbb{R}}_{+}^{q}$. Then $w$ is nonnegative if $w_{i}(z) \geq 0$ for all $i=1, \ldots, q$ and $z \in \overline{\mathbb{R}}_{+}^{q}$.

Note that if $w(z)=W z$, where $W \in \mathbb{R}^{q \times q}$, then $w(\cdot)$ is nonnegative if and only if $W$ is a nonnegative matrix.

Proposition $2.3[12]$. Suppose that $\overline{\mathbb{R}}_{+}^{q} \subset \mathcal{V}$. Then $\overline{\mathbb{R}}_{+}^{q}$ is an invariant set with respect to

$$
z(k+1)=w(z(k)), \quad z(0)=z_{0}, \quad k \in \overline{\mathbb{Z}}_{+},
$$

where $z_{0} \in \overline{\mathbb{R}}_{+}^{q}$, if and only if $w: \mathscr{V} \rightarrow \mathbb{R}^{q}$ is nonnegative. 
The following definition introduces several types of stability for the discrete-time nonnegative dynamical system (2.1).

Definition 2.4. The equilibrium solution $z(k) \equiv z_{\mathrm{e}}$ of $(2.1)$ is Lyapunov stable if, for every $\varepsilon>0$, there exists $\delta=\delta(\varepsilon)>0$ such that if $z_{0} \in \mathscr{B}_{\delta}\left(z_{\mathrm{e}}\right) \cap \overline{\mathbb{R}}_{+}^{q}$, then $z(k) \in \mathscr{B}_{\varepsilon}\left(z_{\mathrm{e}}\right) \cap$ $\overline{\mathbb{R}}_{+}^{q}, k \in \overline{\mathbb{Z}}_{+}$. The equilibrium solution $z(k) \equiv z_{\mathrm{e}}$ of $(2.1)$ is semistable if it is Lyapunov stable and there exists $\delta>0$ such that if $z_{0} \in \mathscr{B}_{\delta}\left(z_{\mathrm{e}}\right) \cap \overline{\mathbb{R}}_{+}^{q}$, then $\lim _{k \rightarrow \infty} z(k)$ exists and corresponds to a Lyapunov stable equilibrium point. The equilibrium solution $z(k) \equiv z_{\mathrm{e}}$ of (2.1) is asymptotically stable if it is Lyapunov stable and there exists $\delta>0$ such that if $z_{0} \in \mathscr{B}_{\delta}\left(z_{\mathrm{e}}\right) \cap \overline{\mathbb{R}}_{+}^{q}$, then $\lim _{k \rightarrow \infty} z(k)=z_{\mathrm{e}}$. Finally, the equilibrium solution $z(k) \equiv z_{\mathrm{e}}$ of (2.1) is globally asymptotically stable if the previous statement holds for all $z_{0} \in \overline{\mathbb{R}}_{+}^{q}$.

Finally, recall that a matrix $W \in \mathbb{R}^{q \times q}$ is semistable if and only if $\lim _{k \rightarrow \infty} W^{k}$ exists [12], while $W$ is asymptotically stable if and only if $\lim _{k \rightarrow \infty} W^{k}=0$.

\section{Thermodynamic modeling for discrete-time systems}

3.1. Conservation of energy and the first law of thermodynamics. The fundamental and unifying concept in the analysis of complex (large-scale) dynamical systems is the concept of energy. The energy of a state of a dynamical system is the measure of its ability to produce changes (motion) in its own system state as well as changes in the system states of its surroundings. These changes occur as a direct consequence of the energy flow between different subsystems within the dynamical system. Since heat (energy) is a fundamental concept of thermodynamics involving the capacity of hot bodies (more energetic subsystems) to produce work, thermodynamics is a theory of large-scale dynamical systems [13]. As in thermodynamic systems, dynamical systems can exhibit energy (due to friction) that becomes unavailable to do useful work. This is in turn contributes to an increase in system entropy; a measure of the tendency of a system to lose the ability to do useful work.

To develop discrete-time compartmental models that are consistent with thermodynamic principles, consider the discrete-time large-scale dynamical system $\mathscr{G}$ shown in Figure 3.1 involving $q$ interconnected subsystems. Let $E_{i}: \overline{\mathbb{Z}}_{+} \rightarrow \overline{\mathbb{R}}_{+}$denote the energy (and hence a nonnegative quantity) of the $i$ th subsystem, let $S_{i}: \overline{\mathbb{Z}}_{+} \rightarrow \mathbb{R}$ denote the external energy supplied to (or extracted from) the $i$ th subsystem, let $\sigma_{i j}: \overline{\mathbb{R}}_{+}^{q} \rightarrow \overline{\mathbb{R}}_{+}, i \neq j$, $i, j=1, \ldots, q$, denote the exchange of energy from the $j$ th subsystem to the $i$ th subsystem, and let $\sigma_{i i}: \overline{\mathbb{R}}_{+}^{q} \rightarrow \overline{\mathbb{R}}_{+}, i=1, \ldots, q$, denote the energy loss from the $i$ th subsystem. An energy balance equation for the $i$ th subsystem yields

$$
\Delta E_{i}(k)=\sum_{j=1, j \neq i}^{q}\left[\sigma_{i j}(E(k))-\sigma_{j i}(E(k))\right]-\sigma_{i i}(E(k))+S_{i}(k), \quad k \geq k_{0},
$$

or, equivalently, in vector form,

$$
E(k+1)=w(E(k))-d(E(k))+S(k), \quad k \geq k_{0},
$$




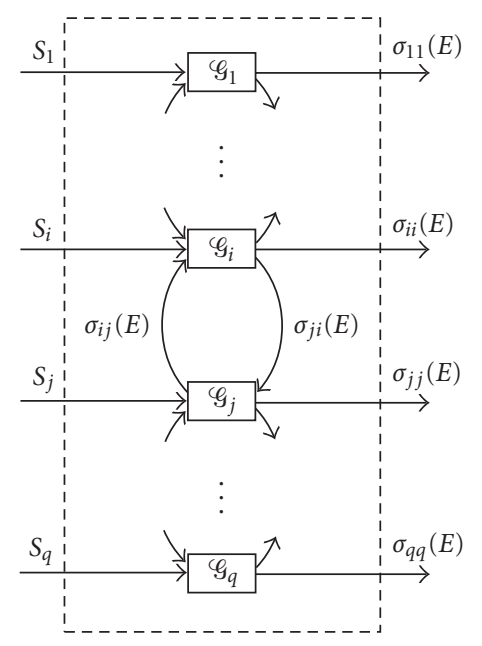

Figure 3.1. Large-scale dynamical system $\mathscr{G}$.

where $E(k)=\left[E_{1}(k), \ldots, E_{q}(k)\right]^{\mathrm{T}}, S(k)=\left[S_{1}(k), \ldots, S_{q}(k)\right]^{\mathrm{T}}, d(E(k))=\left[\sigma_{11}(E(k)), \ldots\right.$, $\left.\sigma_{q q}(E(k))\right]^{\mathrm{T}}, k \geq k_{0}$, and $w=\left[w_{1}, \ldots, w_{q}\right]^{\mathrm{T}}: \overline{\mathbb{R}}_{+}^{q} \rightarrow \mathbb{R}^{q}$ is such that

$$
w_{i}(E)=E_{i}+\sum_{j=1, j \neq i}^{q}\left[\sigma_{i j}(E)-\sigma_{j i}(E)\right], \quad E \in \overline{\mathbb{R}}_{+}^{q} .
$$

Equation (3.1) yields a conservation of energy equation and implies that the change of energy stored in the $i$ th subsystem is equal to the external energy supplied to (or extracted from) the $i$ th subsystem plus the energy gained by the $i$ th subsystem from all other subsystems due to subsystem coupling minus the energy dissipated from the $i$ th subsystem. Note that (3.2) or, equivalently, (3.1) is a statement reminiscent of the first law of thermodynamics for each of the subsystems, with $E_{i}(\cdot), S_{i}(\cdot), \sigma_{i j}(\cdot), i \neq j$, and $\sigma_{i i}(\cdot), i=1, \ldots, q$, playing the role of the $i$ th subsystem internal energy, energy supplied to (or extracted from) the $i$ th subsystem, the energy exchange between subsystems due to coupling, and the energy dissipated to the environment, respectively.

To further elucidate that (3.2) is essentially the statement of the principle of the conservation of energy, let the total energy in the discrete-time large-scale dynamical system $\mathscr{G}$ be given by $U \triangleq \mathbf{e}^{\mathrm{T}} E, E \in \overline{\mathbb{R}}_{+}^{q}$, where $\mathrm{e}^{\mathrm{T}} \triangleq[1, \ldots, 1]$, and let the energy received by the discrete-time large-scale dynamical system $\mathscr{G}$ (in forms other than work) over the discrete-time interval $\left\{k_{1}, \ldots, k_{2}\right\}$ be given by $Q \triangleq \sum_{k=k_{1}}^{k_{2}} \mathbf{e}^{\mathrm{T}}[S(k)-d(E(k))]$, where $E(k)$, $k \geq k_{0}$, is the solution to (3.2). Then, premultiplying (3.2) by $\mathbf{e}^{\mathrm{T}}$ and using the fact that $\mathbf{e}^{\mathrm{T}} w(E) \equiv \mathbf{e}^{\mathrm{T}} E$, it follows that

$$
\Delta U=Q
$$


where $\Delta U \triangleq U\left(k_{2}\right)-U\left(k_{1}\right)$ denotes the variation in the total energy of the discrete-time large-scale dynamical system $\mathscr{G}$ over the discrete-time interval $\left\{k_{1}, \ldots, k_{2}\right\}$. This is a statement of the first law of thermodynamics for the discrete-time large-scale dynamical system $\mathscr{G}$ and gives a precise formulation of the equivalence between variation in system internal energy and heat.

It is important to note that our discrete-time large-scale dynamical system model does not consider work done by the system on the environment nor work done by the environment on the system. Hence, $Q$ can be interpreted physically as the amount of energy that is received by the system in forms other than work. The extension of addressing work performed by and on the system can be easily handled by including an additional state equation, coupled to the energy balance equation (3.2), involving volume states for each subsystem [13]. Since this slight extension does not alter any of the results of the paper, it is not considered here for simplicity of exposition.

For our large-scale dynamical system model $\mathscr{G}$, we assume that $\sigma_{i j}(E)=0, E \in \overline{\mathbb{R}}_{+}^{q}$, whenever $E_{j}=0, i, j=1, \ldots, q$. This constraint implies that if the energy of the $j$ th subsystem of $\mathscr{G}$ is zero, then this subsystem cannot supply any energy to its surroundings nor dissipate energy to the environment. Furthermore, for the remainder of this paper, we assume that $E_{i} \geq \sigma_{i i}(E)-S_{i}-\sum_{j=1, j \neq i}^{q}\left[\sigma_{i j}(E)-\sigma_{j i}(E)\right]=-\Delta E_{i}, E \in \overline{\mathbb{R}}_{+}^{q}, S \in \mathbb{R}^{q}, i=1, \ldots, q$. This constraint implies that the energy that can be dissipated, extracted, or exchanged by the $i$ th subsystem cannot exceed the current energy in the subsystem. Note that this assumption implies that $E(k) \geq \geq 0$ for all $k \geq k_{0}$.

Next, premultiplying (3.2) by $\mathbf{e}^{\mathrm{T}}$ and using the fact that $\mathbf{e}^{\mathrm{T}} w(E) \equiv \mathbf{e}^{\mathrm{T}} E$, it follows that

$$
\mathbf{e}^{\mathrm{T}} E\left(k_{1}\right)=\mathbf{e}^{\mathrm{T}} E\left(k_{0}\right)+\sum_{k=k_{0}}^{k_{1}-1} \mathbf{e}^{\mathrm{T}} S(k)-\sum_{k=k_{0}}^{k_{1}-1} \mathbf{e}^{\mathrm{T}} d(E(k)), \quad k_{1} \geq k_{0} .
$$

Now, for the discrete-time large-scale dynamical system $\mathscr{G}$, define the input $u(k) \triangleq S(k)$ and the output $y(k) \triangleq d(E(k))$. Hence, it follows from (3.5) that the discrete-time largescale dynamical system $\mathscr{G}$ is lossless [23] with respect to the energy supply rate $r(u, y)=$ $\mathbf{e}^{\mathrm{T}} u-\mathbf{e}^{\mathrm{T}} y$ and with the energy storage function $U(E) \triangleq \mathbf{e}^{\mathrm{T}} E, E \in \overline{\mathbb{R}}_{+}^{q}$. This implies that (see [23] for details)

$$
0 \leq U_{\mathrm{a}}\left(E_{0}\right)=U\left(E_{0}\right)=U_{\mathrm{r}}\left(E_{0}\right)<\infty, \quad E_{0} \in \overline{\mathbb{R}}_{+}^{q},
$$

where

$$
\begin{gathered}
U_{\mathrm{a}}\left(E_{0}\right) \triangleq-\inf _{u(\cdot), K \geq k_{0}} \sum_{k=k_{0}}^{K-1}\left(\mathbf{e}^{\mathrm{T}} u(k)-\mathbf{e}^{\mathrm{T}} y(k)\right), \\
U_{\mathrm{r}}\left(E_{0}\right) \triangleq \inf _{u(\cdot), K \geq-k_{0}+1} \sum_{k=-K}^{k_{0}-1}\left(\mathbf{e}^{\mathrm{T}} u(k)-\mathbf{e}^{\mathrm{T}} y(k)\right),
\end{gathered}
$$

and $E_{0}=E\left(k_{0}\right) \in \overline{\mathbb{R}}_{+}^{q}$. Since $U_{\mathrm{a}}\left(E_{0}\right)$ is the maximum amount of stored energy which can be extracted from the discrete-time large-scale dynamical system $\mathcal{G}_{\text {at }}$ any discrete-time instant $K$, and $U_{\mathrm{r}}\left(E_{0}\right)$ is the minimum amount of energy which can be delivered to 
the discrete-time large-scale dynamical system $G_{S}$ to transfer it from a state of minimum potential $E(-K)=0$ to a given state $E\left(k_{0}\right)=E_{0}$, it follows from (3.6) that the discretetime large-scale dynamical system $\mathscr{G}$ can deliver to its surroundings all of its stored subsystem energies and can store all of the work done to all of its subsystems. In the case where $S(k) \equiv 0$, it follows from (3.5) and the fact that $\sigma_{i i}(E) \geq 0, E \in \overline{\mathbb{R}}_{+}^{q}, i=1, \ldots, q$, that the zero solution $E(k) \equiv 0$ of the discrete-time large-scale dynamical system $\mathscr{G}$ with the energy balance equation (3.2) is Lyapunov stable with Lyapunov function $U(E)$ corresponding to the total energy in the system.

The next result shows that the large-scale dynamical system $\mathscr{G}$ is locally controllable.

Proposition 3.1. Consider the discrete-time large-scale dynamical system $G$ with energy balance equation (3.2). Then for every equilibrium state $E_{\mathrm{e}} \in \overline{\mathbb{R}}_{+}^{q}$ and every $\varepsilon>0$ and $T \in$ $\mathbb{Z}_{+}$, there exist $S_{\mathrm{e}} \in \mathbb{R}^{q}, \alpha>0$, and $\hat{T} \in\{0, \ldots, T\}$ such that for every $\hat{E} \in \overline{\mathbb{R}}_{+}^{q}$ with $\| \hat{E}-$ $E_{\mathrm{e}} \| \leq \alpha T$, there exists $S:\{0, \ldots, \hat{T}\} \rightarrow \mathbb{R}^{q}$ such that $\left\|S(k)-S_{\mathrm{e}}\right\| \leq \mathcal{E}, k \in\{0, \ldots, \hat{T}\}$, and $E(k)=E_{\mathrm{e}}+\left(\left(\hat{E}-E_{\mathrm{e}}\right) / \widehat{T}\right) k, k \in\{0, \ldots, \hat{T}\}$.

Proof. Note that with $S_{\mathrm{e}}=d\left(E_{\mathrm{e}}\right)-w\left(E_{\mathrm{e}}\right)+E_{\mathrm{e}}$, the state $E_{\mathrm{e}} \in \overline{\mathbb{R}}_{+}^{q}$ is an equilibrium state of (3.2). Let $\theta>0$ and $T \in \mathbb{Z}_{+}$, and define

$$
M(\theta, T) \triangleq \sup _{E \in \overline{\mathscr{S}}_{1}(0), k \in\{0, \ldots, T\}}\left\|w\left(E_{\mathrm{e}}+k \theta E\right)-w\left(E_{\mathrm{e}}\right)-d\left(E_{\mathrm{e}}+k \theta E\right)+d\left(E_{\mathrm{e}}\right)-k \theta E\right\| .
$$

Note that for every $T \in \mathbb{Z}_{+}, \lim _{\theta \rightarrow 0^{+}} M(\theta, T)=0$. Next, let $\varepsilon>0$ and $T \in \mathbb{Z}_{+}$be given, and let $\alpha>0$ be such that $M(\alpha, T)+\alpha \leq \varepsilon$. (The existence of such an $\alpha$ is guaranteed since $M(\alpha, T) \rightarrow 0$ as $\alpha \rightarrow 0^{+}$.) Now, let $\hat{E} \in \overline{\mathbb{R}}_{+}^{q}$ be such that $\left\|\hat{E}-E_{\mathrm{e}}\right\| \leq \alpha T$. With $\hat{T} \triangleq$ $\left\lceil\left\|\widehat{E}-E_{\mathrm{e}}\right\| / \alpha\right\rceil \leq T$, where $\lceil x\rceil$ denotes the smallest integer greater than or equal to $x$, and

$$
S(k)=-w(E(k))+d(E(k))+E(k)+\frac{\widehat{E}-E_{\mathrm{e}}}{\left\lceil|| \hat{E}-E_{\mathrm{e}}|| / \alpha\right\rceil}, \quad k \in\{0, \ldots, \widehat{T}\},
$$

it follows that

$$
E(k)=E_{\mathrm{e}}+\frac{\left(\widehat{E}-E_{\mathrm{e}}\right)}{\left\lceil\left\|\hat{E}-E_{\mathrm{e}}\right\| / \alpha\right\rceil} k, \quad k \in\{0, \ldots, \widehat{T}\}
$$

is a solution to (3.2). The result is now immediate by noting that $E(\widehat{T})=\widehat{E}$ and

$$
\begin{aligned}
\left\|S(k)-S_{\mathrm{e}}\right\| \leq & \| w\left(E_{\mathrm{e}}+\frac{\left(\widehat{E}-E_{\mathrm{e}}\right)}{\left\lceil|| \widehat{E}-E_{\mathrm{e}} \| / \alpha\right\rceil} k\right)-w\left(E_{\mathrm{e}}\right)-d\left(E_{\mathrm{e}}+\frac{\left(\widehat{E}-E_{\mathrm{e}}\right)}{\left\lceil\left\|\widehat{E}-E_{\mathrm{e}}\right\| / \alpha\right\rceil} k\right) \\
& +d\left(E_{\mathrm{e}}\right)-\frac{\left(\widehat{E}-E_{\mathrm{e}}\right)}{\left\lceil\left\|\widehat{E}-E_{\mathrm{e}}\right\| / \alpha\right\rceil} k \|+\alpha \\
\leq & M(\alpha, T)+\alpha \\
\leq & \varepsilon, \quad k \in\{0, \ldots, \widehat{T}\} .
\end{aligned}
$$

It follows from Proposition 3.1 that the discrete-time large-scale dynamical system $\mathscr{G}$ with the energy balance equation (3.2) is reachable from and controllable to the origin in 
$\overline{\mathbb{R}}_{+}^{q}$. Recall that the discrete-time large-scale dynamical system $\varphi$ with the energy balance equation (3.2) is reachable from the origin in $\overline{\mathbb{R}}_{+}^{q}$ if, for all $E_{0}=E\left(k_{0}\right) \in \overline{\mathbb{R}}_{+}^{q}$, there exist a finite time $k_{\mathrm{i}} \leq k_{0}$ and an input $S(k)$ defined on $\left\{k_{\mathrm{i}}, \ldots, k_{0}\right\}$ such that the state $E(k), k \geq k_{\mathrm{i}}$, can be driven from $E\left(k_{\mathrm{i}}\right)=0$ to $E\left(k_{0}\right)=E_{0}$. Alternatively, $\mathscr{G}$ is controllable to the origin in $\overline{\mathbb{R}}_{+}^{q}$ if, for all $E_{0}=E\left(k_{0}\right) \in \overline{\mathbb{R}}_{+}^{q}$, there exist a finite time $k_{\mathrm{f}} \geq k_{0}$ and an input $S(k)$ defined on $\left\{k_{0}, \ldots, k_{\mathrm{f}}\right\}$ such that the state $E(k), k \geq k_{0}$, can be driven from $E\left(k_{0}\right)=E_{0}$ to $E\left(k_{\mathrm{f}}\right)=0$. We let $\boldsymbol{U}_{\mathrm{r}}$ denote the set of all admissible bounded energy inputs to the discrete-time large-scale dynamical system $\mathscr{G}_{\mathrm{S}}$ such that for any $K \geq-k_{0}$, the system energy state can be driven from $E(-K)=0$ to $E\left(k_{0}\right)=E_{0} \in \overline{\mathbb{R}}_{+}^{q}$ by $S(\cdot) \in \mathcal{u}_{\mathrm{r}}$, and we let $\boldsymbol{u}_{\mathrm{c}}$ denote the set of all admissible bounded energy inputs to the discrete-time large-scale dynamical

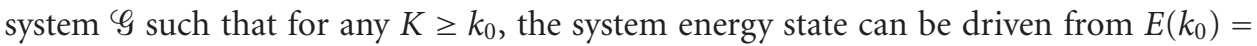
$E_{0} \in \overline{\mathbb{R}}_{+}^{q}$ to $E(K)=0$ by $S(\cdot) \in \mathcal{U}_{\mathrm{c}}$. Furthermore, let $\mathcal{U}$ be an input space that is a subset of bounded continuous $\mathbb{R}^{q}$-valued functions on $\mathbb{Z}$. The spaces $\boldsymbol{U}_{\mathrm{r}}, \boldsymbol{U}_{\mathrm{c}}$, and $\boldsymbol{u}$ are assumed to be closed under the shift operator; that is, if $S(\cdot) \in \mathcal{U}$ (resp., $\boldsymbol{U}_{\mathrm{c}}$ or $\boldsymbol{U}_{\mathrm{r}}$ ), then the function $S_{K}$ defined by $S_{K}(k)=S(k+K)$ is contained in $\boldsymbol{u}$ (resp., $\boldsymbol{U}_{\mathrm{c}}$ or $\boldsymbol{U}_{\mathrm{r}}$ ) for all $K \geq 0$.

3.2. Nonconservation of entropy and the second law of thermodynamics. The nonlinear energy balance equation (3.2) can exhibit a full range of nonlinear behavior including bifurcations, limit cycles, and even chaos. However, a thermodynamically consistent energy flow model should ensure that the evolution of the system energy is diffusive (parabolic) in character with convergent subsystem energies. Hence, to ensure a thermodynamically consistent energy flow model, we require the following axioms. For the statement of these axioms, we first recall the following graph-theoretic notions.

Definition $3.2[2]$. A directed graph $G(\mathscr{C})$ associated with the connectivity matrix $\mathscr{C} \in \mathbb{R}^{q \times q}$ has vertices $\{1,2, \ldots, q\}$ and an $\operatorname{arc}$ from vertex $i$ to vertex $j, i \neq j$, if and only if $\mathscr{C}_{(j, i)} \neq 0$. A graph $G(\mathscr{C})$ associated with the connectivity matrix $\mathscr{C} \in \mathbb{R}^{q \times q}$ is a directed graph for which the arc set is symmetric; that is, $\mathscr{C}=\mathscr{C}^{\mathrm{T}}$. It is said that $G(\mathscr{C})$ is strongly connected if for any ordered pair of vertices $(i, j), i \neq j$, there exists a path (i.e., sequence of arcs) leading from $i$ to $j$.

Recall that $\mathscr{C} \in \mathbb{R}^{q \times q}$ is irreducible; that is, there does not exist a permutation matrix such that $\mathscr{C}$ is cogredient to a lower-block triangular matrix, if and only if $G(\mathscr{C})$ is strongly connected (see [2, Theorem 2.7]). Let $\phi_{i j}(E) \triangleq \sigma_{i j}(E)-\sigma_{j i}(E), E \in \overline{\mathbb{R}}_{+}^{q}$, denote the net energy exchange between subsystems $\mathscr{G}_{i}$ and $\mathscr{G}_{j}$ of the discrete-time large-scale dynamical system $\mathscr{G}$.

Aхіом 1. For the connectivity matrix $\mathscr{b} \in \mathbb{R}^{q \times q}$ associated with the large-scale dynamical system $G$ defined by

$$
\begin{gathered}
\mathscr{C}_{(i, j)}=\left\{\begin{array}{ll}
0 & \text { if } \phi_{i j}(E) \equiv 0, \\
1 & \text { otherwise, }
\end{array} \quad i \neq j, i, j=1, \ldots, q,\right. \\
\mathscr{C}_{(i, i)}=-\sum_{k=1, k \neq i}^{q} \mathscr{C}_{(k, i)}, \quad i=j, i=1, \ldots, q,
\end{gathered}
$$

$\operatorname{rank} \mathscr{C}=q-1$, and for $\mathscr{C}_{(i, j)}=1, i \neq j, \phi_{i j}(E)=0$ if and only if $E_{i}=E_{j}$. 
Aхіом 2. For $i, j=1, \ldots, q,\left(E_{i}-E_{j}\right) \phi_{i j}(E) \leq 0, E \in \overline{\mathbb{R}}_{+}^{q}$.

Axıом 3. For $i, j=1, \ldots, q,\left(\Delta E_{i}-\Delta E_{j}\right) /\left(E_{i}-E_{j}\right) \geq-1, E_{i} \neq E_{j}$.

The fact that $\phi_{i j}(E)=0$ if and only if $E_{i}=E_{j}, i \neq j$, implies that subsystems $\varphi_{i}$ and $\mathscr{G}_{j}$ of $\mathscr{G}_{S}$ are connected; alternatively, $\phi_{i j}(E) \equiv 0$ implies that $\mathscr{G}_{i}$ and $\mathscr{G}_{j}$ are disconnected. Axiom 1 implies that if the energies in the connected subsystems $\mathscr{G}_{i}$ and $\mathscr{G}_{j}$ are equal, then energy exchange between these subsystems is not possible. This is a statement consistent with the zeroth law of thermodynamics which postulates that temperature equality is a necessary and sufficient condition for thermal equilibrium. Furthermore, it follows from the fact that $\mathscr{C}=\mathscr{C}^{\mathrm{T}}$ and $\operatorname{rank} \mathscr{C}=q-1$ that the connectivity matrix $\mathscr{C}$ is irreducible which implies that for any pair of subsystems $\mathscr{G}_{i}$ and $\mathscr{G}_{j}, i \neq j$, of $\mathscr{G}$, there exists a sequence of connected subsystems of $\mathscr{G}_{\text {that }}$ connect $\mathscr{G}_{i}$ and $\mathscr{G}_{j}$. Axiom 2 implies that energy is exchanged from more energetic subsystems to less energetic subsystems and is consistent with the second law of thermodynamics which states that heat (energy) must flow in the direction of lower temperatures. Furthermore, note that $\phi_{i j}(E)=-\phi_{j i}(E), E \in \overline{\mathbb{R}}_{+}^{q}, i \neq j$, $i, j=1, \ldots, q$, which implies conservation of energy between lossless subsystems. With $S(k) \equiv 0$, Axioms 1 and 2 along with the fact that $\phi_{i j}(E)=-\phi_{j i}(E), E \in \overline{\mathbb{R}}_{+}^{q}, i \neq j, i, j=$ $1, \ldots, q$, imply that at a given instant of time, energy can only be transported, stored, or dissipated but not created and the maximum amount of energy that can be transported and/or dissipated from a subsystem cannot exceed the energy in the subsystem. Finally, Axiom 3 implies that for any pair of connected subsystems $\mathscr{G}_{i}$ and $\mathscr{G}_{j}, i \neq j$, the energy difference between consecutive time instants is monotonic; that is, $\left[E_{i}(k+1)-E_{j}(k+\right.$ $1)]\left[E_{i}(k)-E_{j}(k)\right] \geq 0$ for all $E_{i} \neq E_{j}, k \geq k_{0}, i, j=1, \ldots, q$.

Next, we establish a Clausius-type inequality for our thermodynamically consistent energy flow model.

Proposition 3.3. Consider the discrete-time large-scale dynamical system $\mathscr{G}$ with energy balance equation (3.2) and assume that Axioms 1, 2, and 3 hold. Then for all $E_{0} \in \overline{\mathbb{R}}_{+}^{q}$, $k_{\mathrm{f}} \geq k_{0}$, and $S(\cdot) \in \mathcal{U}$ such that $E\left(k_{\mathrm{f}}\right)=E\left(k_{0}\right)=E_{0}$,

$$
\begin{aligned}
& \sum_{k=k_{0}}^{k_{\mathrm{f}}-1} \sum_{i=1}^{q} \frac{S_{i}(k)-\sigma_{i i}(E(k))}{c+E_{i}(k+1)} \\
&=\sum_{k=k_{0}}^{k_{\mathrm{f}}-1} \sum_{i=1}^{q} \frac{Q_{i}(k)}{c+E_{i}(k+1)} \leq 0,
\end{aligned}
$$

where $c>0, Q_{i}(k) \triangleq S_{i}(k)-\sigma_{i i}(E(k)), i=1, \ldots, q$, is the amount of net energy (heat) received by the ith subsystem at the $k$ th instant, and $E(k), k \geq k_{0}$, is the solution to (3.2) with initial condition $E\left(k_{0}\right)=E_{0}$. Furthermore, equality holds in (3.13) if and only if $\Delta E_{i}(k)=0$, $i=1, \ldots, q$, and $E_{i}(k)=E_{j}(k), i, j=1, \ldots, q, i \neq j, k \in\left\{k_{0}, \ldots, k_{\mathrm{f}}-1\right\}$.

Proof. Since $E(k) \geq \geq 0, k \geq k_{0}$, and $\phi_{i j}(E)=-\phi_{j i}(E), E \in \overline{\mathbb{R}}_{+}^{q}, i \neq j, i, j=1, \ldots, q$, it follows from (3.2), Axioms 2 and 3 , and the fact that $x /(x+1) \leq \log _{e}(1+x), x>-1$ 
that

$$
\begin{aligned}
\sum_{k=k_{0}}^{k_{\mathrm{f}}-1} \sum_{i=1}^{q} \frac{Q_{i}(k)}{c+E_{i}(k+1)}= & \sum_{k=k_{0}}^{k_{\mathrm{f}}-1} \sum_{i=1}^{q} \frac{\Delta E_{i}(k)-\sum_{j=1, j \neq i}^{q} \phi_{i j}(E(k))}{c+E_{i}(k+1)} \\
= & \sum_{k=k_{0}}^{k_{\mathrm{f}}-1} \sum_{i=1}^{q}\left[\frac{\Delta E_{i}(k)}{c+E_{i}(k)}\right]\left[1+\frac{\Delta E_{i}(k)}{c+E_{i}(k)}\right]^{-1} \\
& -\sum_{k=k_{0}}^{k_{\mathrm{f}}-1} \sum_{i=1}^{q} \sum_{j=1, j \neq i}^{q} \frac{\phi_{i j}(E(k))}{c+E_{i}(k+1)} \\
\leq & \sum_{i=1}^{q} \log _{e}\left(\frac{c+E_{i}\left(k_{\mathrm{f}}\right)}{c+E_{i}\left(k_{0}\right)}\right)-\sum_{k=k_{0}} \sum_{i=1}^{q} \sum_{j=1, j \neq i}^{q} \frac{\phi_{i j}(E(k))}{c+E_{i}(k+1)} \\
= & -\sum_{k=k_{0}}^{k_{\mathrm{f}}-1} \sum_{i=1}^{q-1} \sum_{j=i+1}^{q}\left(\frac{\phi_{i j}(E(k))}{c+E_{i}(k+1)}-\frac{\phi_{i j}(E(k))}{c+E_{j}(k+1)}\right) \\
= & -\sum_{k=k_{0}}^{k_{\mathrm{f}}-1} \sum_{i=1}^{q-1} \sum_{j=i+1}^{q} \frac{\phi_{i j}(E(k))\left(E_{j}(k+1)-E_{i}(k+1)\right)}{\left(c+E_{i}(k+1)\right)\left(c+E_{j}(k+1)\right)} \\
\leq & 0,
\end{aligned}
$$

which proves (3.13).

Alternatively, equality holds in (3.13) if and only if $\sum_{k=k_{0}}^{k_{\mathrm{f}}-1}\left(\Delta E_{i}(k) /\left(c+E_{i}(k+1)\right)\right)=0$, $i=1, \ldots, q$, and $\phi_{i j}(E(k))\left(E_{j}(k+1)-E_{i}(k+1)\right)=0, i, j=1, \ldots, q, i \neq j, k \geq k_{0}$. Moreover, $\sum_{k=k_{0}}^{k_{\mathrm{f}}-1}\left(\Delta E_{i}(k) /\left(c+E_{i}(k+1)\right)\right)=0$ is equivalent to $\Delta E_{i}(k)=0, i=1, \ldots, q, k \in\left\{k_{0}, \ldots, k_{\mathrm{f}}-\right.$ $1\}$. Hence, $\phi_{i j}(E(k))\left(E_{j}(k+1)-E_{i}(k+1)\right)=\phi_{i j}(E(k))\left(E_{j}(k)-E_{i}(k)\right)=0, i, j=1, \ldots, q$, $i \neq j, k \geq k_{0}$. Thus, it follows from Axioms 1, 2, and 3 that equality holds in (3.13) if and only if $\Delta E_{i}=0, i=1, \ldots, q$, and $E_{j}=E_{i}, i, j=1, \ldots, q, i \neq j$.

Inequality (3.13) is analogous to Clausius' inequality for reversible and irreversible thermodynamics as applied to discrete-time large-scale dynamical systems. It follows from Axiom 1 and (3.2) that for the isolated discrete-time large-scale dynamical system $\varphi$; that is, $S(k) \equiv 0$ and $d(E(k)) \equiv 0$, the energy states given by $E_{\mathrm{e}}=\alpha \mathbf{e}, \alpha \geq 0$, correspond to the equilibrium energy states of $\mathscr{G}$. Thus, we can define an equilibrium process as a process where the trajectory of the discrete-time large-scale dynamical system $\mathscr{G}$ stays at the equilibrium point of the isolated system $\mathscr{G}$. The input that can generate such a trajectory can be given by $S(k)=d(E(k)), k \geq k_{0}$. Alternatively, a nonequilibrium process is a process that is not an equilibrium one. Hence, it follows from Axiom 1 that for an equilibrium process, $\phi_{i j}(E(k)) \equiv 0, k \geq k_{0}, i \neq j, i, j=1, \ldots, q$, and thus, by Proposition 3.3 and $\Delta E_{i}=0$, $i=1, \ldots, q$, inequality (3.13) is satisfied as an equality. Alternatively, for a nonequilibrium process, it follows from Axioms 1, 2, and 3 that (3.13) is satisfied as a strict inequality.

Next, we give a deterministic definition of entropy for the discrete-time large-scale dynamical system $\mathscr{G}$ that is consistent with the classical thermodynamic definition of entropy. 
Definition 3.4. For the discrete-time large-scale dynamical system $\mathscr{G}$ with energy balance equation (3.2), a function $\mathscr{Y}: \overline{\mathbb{R}}_{+}^{q} \rightarrow \mathbb{R}$ satisfying

$$
\mathscr{S}\left(E\left(k_{2}\right)\right) \geq \mathscr{S}\left(E\left(k_{1}\right)\right)+\sum_{k=k_{1}}^{k_{2}-1} \sum_{i=1}^{q} \frac{S_{i}(k)-\sigma_{i i}(E(k))}{c+E_{i}(k+1)},
$$

for any $k_{2} \geq k_{1} \geq k_{0}$ and $S(\cdot) \in \mathcal{U}$, is called the entropy of $\mathscr{G}$.

Next, we show that (3.13) guarantees the existence of an entropy function for $\mathscr{G}$. For this result, define, the available entropy of the large-scale dynamical system $\mathscr{G}$ by

$$
\mathscr{S}_{\mathrm{a}}\left(E_{0}\right) \triangleq-\sup _{S(\cdot) \in \Theta_{\mathrm{c}}, K \geq k_{0}} \sum_{k=k_{0}}^{K-1} \sum_{i=1}^{q} \frac{S_{i}(k)-\sigma_{i i}(E(k))}{c+E_{i}(k+1)},
$$

where $E\left(k_{0}\right)=E_{0} \in \overline{\mathbb{R}}_{+}^{q}$ and $E(K)=0$, and define the required entropy supply of the largescale dynamical system $\mathscr{G}$ by

$$
\mathscr{Y}_{\mathrm{r}}\left(E_{0}\right) \triangleq \sup _{S(\cdot) \in U_{\mathrm{r}}, K \geq-k_{0}+1} \sum_{k=-K}^{k_{0}-1} \sum_{i=1}^{q} \frac{S_{i}(k)-\sigma_{i i}(E(k))}{c+E_{i}(k+1)},
$$

where $E(-K)=0$ and $E\left(k_{0}\right)=E_{0} \in \overline{\mathbb{R}}_{+}^{q}$. Note that the available entropy $\mathscr{Y}_{\mathrm{a}}\left(E_{0}\right)$ is the minimum amount of scaled heat (entropy) that can be extracted from the large-scale dynamical system $\mathscr{G}$ in order to transfer it from an initial state $E\left(k_{0}\right)=E_{0}$ to $E(K)=0$. Alternatively, the required entropy supply $\mathscr{S}_{\mathrm{r}}\left(E_{0}\right)$ is the maximum amount of scaled heat (entropy) that can be delivered to $\mathscr{G}$ to transfer it from the origin to a given initial state $E\left(k_{0}\right)=E_{0}$.

Theorem 3.5. Consider the discrete-time large-scale dynamical system $\mathcal{G}$ with energy balance equation (3.2) and assume that Axioms 2 and 3 hold. Then there exists an entropy

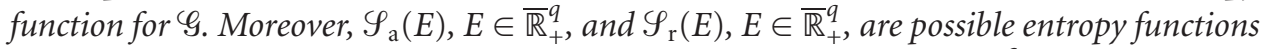
for $\mathscr{G}_{\text {with }} \mathscr{S}_{\mathrm{a}}(0)=\mathscr{Y}_{\mathrm{r}}(0)=0$. Finally, all entropy functions $\mathscr{S}(E), E \in \overline{\mathbb{R}}_{+}^{q}$, for $\mathscr{G}_{\text {satisfy }}$

$$
\mathscr{Y}_{\mathrm{r}}(E) \leq \mathscr{S}(E)-\mathscr{S}(0) \leq \mathscr{Y}_{\mathrm{a}}(E), \quad E \in \overline{\mathbb{R}}_{+}^{q} .
$$

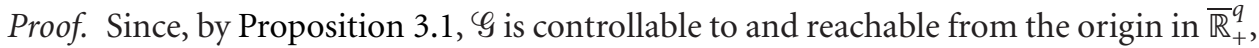
it follows from (3.16) and (3.17) that $\mathscr{S}_{\mathrm{a}}\left(E_{0}\right)<\infty, E_{0} \in \overline{\mathbb{R}}_{+}^{q}$, and $\mathscr{S}_{\mathrm{r}}\left(E_{0}\right)>-\infty, E_{0} \in \overline{\mathbb{R}}_{+}^{q}$, respectively. Next, let $E_{0} \in \overline{\mathbb{R}}_{+}^{q}$ and let $S(\cdot) \in \mathcal{U}$ be such that $E\left(k_{\mathrm{i}}\right)=E\left(k_{\mathrm{f}}\right)=0$ and $E\left(k_{0}\right)=$ $E_{0}$, where $k_{\mathrm{i}} \leq k_{0} \leq k_{\mathrm{f}}$. In this case, it follows from (3.13) that

$$
\sum_{k=k_{\mathrm{i}}}^{k_{\mathrm{f}}-1} \sum_{i=1}^{q} \frac{S_{i}(k)-\sigma_{i i}(E(k))}{c+E_{i}(k+1)} \leq 0,
$$

or, equivalently,

$$
\sum_{k=k_{\mathrm{i}}}^{k_{0}-1} \sum_{i=1}^{q} \frac{S_{i}(k)-\sigma_{i i}(E(k))}{c+E_{i}(k+1)} \leq-\sum_{k=k_{0}}^{k_{\mathrm{f}}-1} \sum_{i=1}^{q} \frac{S_{i}(k)-\sigma_{i i}(E(k))}{c+E_{i}(k+1)} .
$$


Now, taking the supremum on both sides of (3.20) over all $S(\cdot) \in \boldsymbol{U}_{\mathrm{r}}$ and $k_{\mathrm{i}}+1 \leq k_{0}$, we obtain

$$
\begin{aligned}
\mathscr{S}_{\mathrm{r}}\left(E_{0}\right) & =\sup _{S(\cdot) \in \mathscr{U}_{\mathrm{r}}, k_{\mathrm{i}}+1 \leq k_{0}} \sum_{k=k_{\mathrm{i}}}^{k_{0}-1} \sum_{i=1}^{q} \frac{S_{i}(k)-\sigma_{i i}(E(k))}{c+E_{i}(k+1)} \\
& \leq-\sum_{k=k_{0}}^{k_{\mathrm{f}}-1} \sum_{i=1}^{q} \frac{S_{i}(k)-\sigma_{i i}(E(k))}{c+E_{i}(k+1)} .
\end{aligned}
$$

Next, taking the infimum on both sides of (3.21) over all $S(\cdot) \in U_{\mathrm{c}}$ and $k_{\mathrm{f}} \geq k_{0}$, we obtain $\mathscr{S}_{\mathrm{r}}\left(E_{0}\right) \leq \mathscr{Y}_{\mathrm{a}}\left(E_{0}\right), E_{0} \in \overline{\mathbb{R}}_{+}^{q}$, which implies that $-\infty<\mathscr{S}_{\mathrm{r}}\left(E_{0}\right) \leq \mathscr{Y}_{\mathrm{a}}\left(E_{0}\right)<+\infty, E_{0} \in \overline{\mathbb{R}}_{+}^{q}$. Hence, the function $\mathscr{S}_{\mathrm{a}}(\cdot)$ and $\mathscr{S}_{\mathrm{r}}(\cdot)$ are well defined.

Next, it follows from the definition of $\mathscr{S}_{\mathrm{a}}(\cdot)$ that, for any $K \geq k_{1}$ and $S(\cdot) \in \mathcal{U}_{\mathrm{c}}$ such that $E\left(k_{1}\right) \in \overline{\mathbb{R}}_{+}^{q}$ and $E(K)=0$,

$$
-\mathscr{Y}_{\mathrm{a}}\left(E\left(k_{1}\right)\right) \geq \sum_{k=k_{1}}^{k_{2}-1} \sum_{i=1}^{q} \frac{S_{i}(k)-\sigma_{i i}(E(k))}{c+E_{i}(k+1)}+\sum_{k=k_{2}}^{K-1} \sum_{i=1}^{q} \frac{S_{i}(k)-\sigma_{i i}(E(k))}{c+E_{i}(k+1)}, \quad k_{1} \leq k_{2} \leq K,
$$

and hence

$$
\begin{aligned}
-\mathscr{Y}_{\mathrm{a}}\left(E\left(k_{1}\right)\right) & \geq \sum_{k=k_{1}}^{k_{2}-1} \sum_{i=1}^{q} \frac{S_{i}(k)-\sigma_{i i}(E(k))}{c+E_{i}(k+1)}+\sup _{S(\cdot) \in U_{\mathrm{c}}, K \geq k_{2}} \sum_{k=k_{2}}^{K-1} \sum_{i=1}^{q} \frac{S_{i}(k)-\sigma_{i i}(E(k))}{c+E_{i}(k+1)} \\
& =\sum_{k=k_{1}}^{k_{2}-1} \sum_{i=1}^{q} \frac{S_{i}(k)-\sigma_{i i}(E(k))}{c+E_{i}(k+1)}-\mathscr{S}_{\mathrm{a}}\left(E\left(k_{2}\right)\right),
\end{aligned}
$$

which implies that $\mathscr{Y}_{\mathrm{a}}(E), E \in \overline{\mathbb{R}}_{+}^{q}$, satisfies (3.15). Thus, $\mathscr{S}_{\mathrm{a}}(E), E \in \overline{\mathbb{R}}_{+}^{q}$, is a possible entropy function for $\mathscr{G}$. Note that with $E\left(k_{0}\right)=E(K)=0$, it follows from (3.13) that the supremum in (3.16) is taken over the set of nonpositive values with one of the values being zero for $S(k) \equiv 0$. Thus, $\mathscr{S}_{\mathrm{a}}(0)=0$. Similarly, it can be shown that $\mathscr{S}_{\mathrm{r}}(E), E \in \overline{\mathbb{R}}_{+}^{q}$, given by (3.17) satisfies (3.15), and hence is a possible entropy function for the system $\mathscr{G}$ with $\mathscr{S}_{\mathrm{r}}(0)=0$.

Next, suppose that there exists an entropy function $\mathscr{S}: \overline{\mathbb{R}}_{+}^{q} \rightarrow \mathbb{R}$ for $\mathscr{G}$ and let $E\left(k_{2}\right)=0$ in (3.15). Then it follows from (3.15) that

$$
\mathscr{S}\left(E\left(k_{1}\right)\right)-\mathscr{S}(0) \leq-\sum_{k=k_{1}}^{k_{2}-1} \sum_{i=1}^{q} \frac{S_{i}(k)-\sigma_{i i}(E(k))}{c+E_{i}(k+1)}
$$


for all $k_{2} \geq k_{1}$ and $S(\cdot) \in U_{c}$, which implies that

$$
\begin{aligned}
\mathscr{S}\left(E\left(k_{1}\right)\right)-\mathscr{S}(0) & \leq \inf _{S(\cdot) \in \mathscr{U}_{c}, k_{2} \geq k_{1}}\left[-\sum_{k=k_{1}} \sum_{i=1}^{k_{2}-1} \frac{S_{i}(k)-\sigma_{i i}(E(k))}{c+E_{i}(k+1)}\right] \\
& =-\sup _{S(\cdot) \in U_{c}, k_{2} \geq k_{1}} \sum_{k=k_{1}}^{k_{2}-1} \sum_{i=1}^{q} \frac{S_{i}(k)-\sigma_{i i}(E(k))}{c+E_{i}(k+1)} \\
& =\mathscr{I}_{\mathrm{a}}\left(E\left(k_{1}\right)\right) .
\end{aligned}
$$

Since $E\left(k_{1}\right)$ is arbitrary, it follows that $\mathscr{S}(E)-\mathscr{Y}(0) \leq \mathscr{S}_{\mathrm{a}}(E), E \in \overline{\mathbb{R}}_{+}^{q}$. Alternatively, let $E\left(k_{1}\right)=0$ in (3.15). Then it follows from (3.15) that

$$
\mathscr{S}\left(E\left(k_{2}\right)\right)-\mathscr{S}(0) \geq \sum_{k=k_{1}}^{k_{2}-1} \sum_{i=1}^{q} \frac{S_{i}(k)-\sigma_{i i}(E(k))}{c+E_{i}(k+1)},
$$

for all $k_{1}+1 \leq k_{2}$ and $S(\cdot) \in \mathcal{U}_{\mathrm{r}}$. Hence,

$$
\mathscr{S}\left(E\left(k_{2}\right)\right)-\mathscr{S}(0) \geq \sup _{S(\cdot) \in \mathscr{U}_{\mathrm{r}}, k_{1}+1 \leq k_{2}} \sum_{k=k_{1}}^{k_{2}-1} \sum_{i=1}^{q} \frac{S_{i}(k)-\sigma_{i i}(E(k))}{c+E_{i}(k+1)}=\mathscr{Y}_{\mathrm{r}}\left(E\left(k_{2}\right)\right),
$$

which, since $E\left(k_{2}\right)$ is arbitrary, implies that $\mathscr{S}_{\mathrm{r}}(E) \leq \mathscr{S}(E)-\mathscr{S}(0), E \in \overline{\mathbb{R}}_{+}^{q}$. Thus, all entropy functions for $\mathscr{G}$ satisfy (3.18).

Remark 3.6. It is important to note that inequality (3.13) is equivalent to the existence of an entropy function for $\mathscr{G}$. Sufficiency is simply a statement of Theorem 3.5 while necessity follows from (3.15) with $E\left(k_{2}\right)=E\left(k_{1}\right)$. For nonequilibrium process with energy balance equation (3.2), Definition 3.4 does not provide enough information to define the entropy uniquely. This difficulty has long been pointed out in [19] for thermodynamic systems. A similar remark holds for the definition of ectropy introduced below.

The next proposition gives a closed-form expression for the entropy of $\mathscr{G}$.

Proposition 3.7. Consider the discrete-time large-scale dynamical system $\mathscr{G}$ with energy balance equation (3.2) and assume that Axioms 2 and 3 hold. Then the function $\mathscr{Y}: \overline{\mathbb{R}}_{+}^{q} \rightarrow \mathbb{R}$ given by

$$
\mathscr{S}(E)=\mathbf{e}^{\mathrm{T}} \log _{e}(c \mathbf{e}+E)-q \log _{e} c, \quad E \in \overline{\mathbb{R}}_{+}^{q},
$$

where $c>0$ and $\log _{e}(c \mathbf{e}+E)$ denotes the vector natural logarithm given by $\left[\log _{e}\left(c+E_{1}\right), \ldots\right.$, $\left.\log _{e}\left(c+E_{q}\right)\right]^{T}$, is an entropy function of $G$. 
Proof. Since $E(k) \geq \geq 0, k \geq k_{0}$, and $\phi_{i j}(E)=-\phi_{j i}(E), E \in \overline{\mathbb{R}}_{+}^{q}, i \neq j, i, j=1, \ldots, q$, it follows that

$$
\begin{aligned}
\Delta \mathscr{S}(E(k)) & =\sum_{i=1}^{q} \log _{e}\left[1+\frac{\Delta E_{i}(k)}{c+E_{i}(k)}\right] \\
& \geq \sum_{i=1}^{q}\left[\frac{\Delta E_{i}(k)}{c+E_{i}(k)}\right]\left[1+\frac{\Delta E_{i}(k)}{c+E_{i}(k)}\right]^{-1} \\
& =\sum_{i=1}^{q} \frac{\Delta E_{i}(k)}{c+E_{i}(k)+\Delta E_{i}(k)} \\
& =\sum_{i=1}^{q} \frac{\Delta E_{i}(k)}{c+E_{i}(k+1)} \\
& =\sum_{i=1}^{q}\left[\frac{S_{i}(k)-\sigma_{i i}(E(k))}{c+E_{i}(k+1)}+\sum_{j=1, j \neq i}^{q} \frac{\phi_{i j}(E(k))}{c+E_{i}(k+1)}\right] \\
& =\sum_{i=1}^{q} \frac{S_{i}(k)-\sigma_{i i}(E(k))}{c+E_{i}(k+1)}+\sum_{i=1}^{q-1} \sum_{j=i+1}^{q}\left(\frac{\phi_{i j}(E(k))}{c+E_{i}(k+1)}-\frac{\phi_{i j}(E(k))}{c+E_{j}(k+1)}\right) \\
& =\sum_{i=1}^{q} \frac{S_{i}(k)-\sigma_{i i}(E(k))}{c+E_{i}(k+1)}+\sum_{i=1}^{q-1} \sum_{j=i+1}^{q} \frac{\phi_{i j}(E(k))\left(E_{j}(k+1)-E_{i}(k+1)\right)}{\left(c+E_{i}(k+1)\right)\left(c+E_{j}(k+1)\right)} \\
& \sum_{i}^{q}(k)-\sigma_{i i}(E(k)) \\
c+E_{i}(k+1) & k \geq k_{0},
\end{aligned}
$$

where in (3.29), we used the fact that $\log _{e}(1+x) \geq x /(x+1), x>-1$. Now, summing (3.29) over $\left\{k_{1}, \ldots, k_{2}-1\right\}$ yields (3.15).

Remark 3.8. Note that it follows from the first equality in (3.29) that the entropy function given by (3.28) satisfies (3.15) as an equality for an equilibrium process and as a strict inequality for a nonequilibrium process.

The entropy expression given by (3.28) is identical in form to the Boltzmann entropy for statistical thermodynamics. Due to the fact that the entropy is indeterminate to the extent of an additive constant, we can place the constant $q \log _{e} c$ to zero by taking $c=1$. Since $\mathscr{S}(E)$ given by (3.28) achieves a maximum when all the subsystem energies $E_{i}, i=$ $1, \ldots, q$, are equal, entropy can be thought of as a measure of the tendency of a system to lose the ability to do useful work, lose order, and to settle to a more homogenous state.

3.3. Nonconservation of ectropy. In this subsection, we introduce a new and dual notion to entropy; namely ectropy, describing the status quo of the discrete-time large-scale dynamical system $\mathscr{G}$. First, however, we present a dual inequality to inequality (3.13) that holds for our thermodynamically consistent energy flow model. 
Proposition 3.9. Consider the discrete-time large-scale dynamical system $\mathscr{G}$ with energy balance equation (3.2) and assume that Axioms 1, 2, and 3 hold. Then for all $E_{0} \in \overline{\mathbb{R}}_{+}^{q}$, $k_{\mathrm{f}} \geq k_{0}$, and $S(\cdot) \in \mathcal{U}$ such that $E\left(k_{\mathrm{f}}\right)=E\left(k_{0}\right)=E_{0}$,

$$
\sum_{k=k_{0}}^{k_{\mathrm{f}}-1} \sum_{i=1}^{q} E_{i}(k+1)\left[S_{i}(k)-\sigma_{i i}(E(k))\right]=\sum_{k=k_{0}}^{k_{\mathrm{f}}-1} \sum_{i=1}^{q} E_{i}(k+1) Q_{i}(k) \geq 0,
$$

where $E(k), k \geq k_{0}$, is the solution to (3.2) with initial condition $E\left(k_{0}\right)=E_{0}$. Furthermore, equality holds in (3.30) if and only if $\Delta E_{i}=0$ and $E_{i}=E_{j}, i, j=1, \ldots, q, i \neq j$.

Proof. Since $E(k) \geq \geq 0, k \geq k_{0}$, and $\phi_{i j}(E)=-\phi_{j i}(E), E \in \overline{\mathbb{R}}_{+}^{q}, i \neq j, i, j=1, \ldots, q$, it follows from (3.2) and Axioms 2 and 3 that

$$
\begin{aligned}
2 \sum_{k=k_{0}}^{k_{\mathrm{f}}-1} \sum_{i=1}^{q} E_{i}(k+1) Q_{i}(k)= & \sum_{k=k_{0}} \sum_{i=1}^{k_{\mathrm{f}}-1} E_{i}^{2}(k+1)-E_{i}^{2}(k) \\
& -2 \sum_{k=k_{0}}^{q} \sum_{i=1}^{k_{\mathrm{f}}-1} \sum_{j=1, j \neq i}^{q} E_{i}(k+1) \phi_{i j}(E(k)) \\
& +\sum_{k=k_{0}}^{k_{\mathrm{f}}-1} \sum_{i=1}^{q}\left[\sum_{j=1, j \neq i}^{q} \phi_{i j}(E(k))+S_{i}(k)-\sigma_{i i}(E(k))\right]^{2} \\
= & E^{\mathrm{T}}\left(k_{\mathrm{f}}\right) E\left(k_{\mathrm{f}}\right)-E^{\mathrm{T}}\left(k_{0}\right) E\left(k_{0}\right) \\
& -2 \sum_{k=k_{0}}^{k_{\mathrm{f}}-1} \sum_{i=1}^{q} \sum_{j=1, j \neq i}^{q} E_{i}(k+1) \phi_{i j}(E(k)) \\
& +\sum_{k=k_{0}}^{k_{\mathrm{f}}-1} \sum_{i=1}^{q}\left[\sum_{j=1, j \neq i}^{q} \phi_{i j}(E(k))+S_{i}(k)-\sigma_{i i}(E(k))\right]^{2} \\
= & -2 \sum_{k=k_{0}}^{k_{\mathrm{f}}-1} \sum_{i=1}^{q-1} \sum_{j=i+1}^{q} \phi_{i j}(E(k))\left(E_{i}(k+1)-E_{j}(k+1)\right) \\
& +\sum_{k=k_{0}}^{k_{\mathrm{f}}-1} \sum_{i=1}^{q}\left[\sum_{j=1, j \neq i}^{q} \phi_{i j}(E(k))+S_{i}(k)-\sigma_{i i}(E(k))\right]^{2} \\
\geq & 0,
\end{aligned}
$$

which proves (3.30).

Alternatively, equality holds in (3.30) if and only if $\phi_{i j}(E(k))\left(E_{i}(k+1)-E_{j}(k+1)\right)=0$ and $\sum_{j=1, j \neq i}^{q} \phi_{i j}(E(k))+S_{i}(k)-\sigma_{i i}(E(k))=0, i, j=1, \ldots, q, i \neq j, k \geq k_{0}$. Next, $\sum_{j=1, j \neq i}^{q} \phi_{i j}(E(k))+S_{i}(k)-\sigma_{i i}(E(k))=0$ if and only if $\Delta E_{i}=0, i=1, \ldots, q, k \geq k_{0}$. Hence, $\phi_{i j}(E(k))\left(E_{j}(k+1)-E_{i}(k+1)\right)=\phi_{i j}(E(k))\left(E_{j}(k)-E_{i}(k)\right)=0, i, j=1, \ldots, q, i \neq j, k \geq$ $k_{0}$. Thus, it follows from Axioms 1, 2, and 3 that equality holds in (3.30) if and only if $\Delta E_{i}=0, i=1, \ldots, q$, and $E_{j}=E_{i}, i, j=1, \ldots, q, i \neq j$. 
Note that inequality (3.30) is satisfied as an equality for an equilibrium process and as a strict inequality for a nonequilibrium process. Next, we present the definition of ectropy for the discrete-time large-scale dynamical system $\mathscr{G}$.

Definition 3.10. For the discrete-time large-scale dynamical system $\mathscr{G}$ with energy balance equation (3.2), a function $\mathscr{E}: \overline{\mathbb{R}}_{+}^{q} \rightarrow \mathbb{R}$ satisfying

$$
\mathscr{E}\left(E\left(k_{2}\right)\right) \leq \mathscr{E}\left(E\left(k_{1}\right)\right)+\sum_{k=k_{1}}^{k_{2}-1} \sum_{i=1}^{q} E_{i}(k+1)\left[S_{i}(k)-\sigma_{i i}(E(k))\right]
$$

for any $k_{2} \geq k_{1} \geq k_{0}$ and $S(\cdot) \in \mathcal{U}$, is called the ectropy of $\mathscr{G}$.

For the next result, define the available ectropy of the large-scale dynamical system $\mathscr{G}$ by

$$
\mathscr{E}_{\mathrm{a}}\left(E_{0}\right) \triangleq-\inf _{S(\cdot) \in \mathcal{U}_{\mathrm{c}}, K \geq k_{0}} \sum_{k=k_{0}}^{K-1} \sum_{i=1}^{q} E_{i}(k+1)\left[S_{i}(k)-\sigma_{i i}(E(k))\right],
$$

where $E\left(k_{0}\right)=E_{0} \in \overline{\mathbb{R}}_{+}^{q}$ and $E(K)=0$, and define the required ectropy supply of the largescale dynamical system $\mathscr{G}$ by

$$
\mathscr{E}_{\mathrm{r}}\left(E_{0}\right) \triangleq \inf _{S(\cdot) \in U_{\mathrm{r}}, K \geq-k_{0}+1} \sum_{k=-K}^{k_{0}-1} \sum_{i=1}^{q} E_{i}(k+1)\left[S_{i}(k)-\sigma_{i i}(E(k))\right],
$$

where $E(-K)=0$ and $E\left(k_{0}\right)=E_{0} \in \overline{\mathbb{R}}_{+}^{q}$. Note that the available ectropy $\mathscr{E}_{\mathrm{a}}\left(E_{0}\right)$ is the maximum amount of scaled heat (ectropy) that can be extracted from the large-scale dynamical system $\varphi_{\text {in }}$ order to transfer it from an initial state $E\left(k_{0}\right)=E_{0}$ to $E(K)=0$. Alternatively, the required ectropy supply $\mathscr{E}_{\mathrm{r}}\left(E_{0}\right)$ is the minimum amount of scaled heat (ectropy) that can be delivered to $\mathscr{G}$ to transfer it from an initial state $E(-K)=0$ to a given state $E\left(k_{0}\right)=E_{0}$.

Theorem 3.11. Consider the discrete-time large-scale dynamical system $G$ with energy balance equation (3.2) and assume that Axioms 2 and 3 hold. Then there exists an ectropy function for $\mathscr{G}$. Moreover, $\mathscr{E}_{\mathrm{a}}(E), E \in \overline{\mathbb{R}}_{+}^{q}$, and $\mathscr{E}_{\mathrm{r}}(E), E \in \overline{\mathbb{R}}_{+}^{q}$, are possible ectropy functions for $\mathscr{G}_{\text {with }} \mathscr{E}_{\mathrm{a}}(0)=\mathscr{E}_{\mathrm{r}}(0)=0$. Finally, all ectropy functions $\mathscr{E}(E), E \in \overline{\mathbb{R}}_{+}^{q}$, for $\mathscr{G}_{\text {satisfy }}$

$$
\mathscr{E}_{\mathrm{a}}(E) \leq \mathscr{E}(E)-\mathscr{E}(0) \leq \mathscr{E}_{\mathrm{r}}(E), \quad E \in \overline{\mathbb{R}}_{+}^{q} .
$$

Proof. Since, by Proposition 3.1, $\mathscr{G}$ is controllable to and reachable from the origin in $\overline{\mathbb{R}}_{+}^{q}$, it follows from (3.33) and (3.34) that $\mathscr{E}_{\mathrm{a}}\left(E_{0}\right)>-\infty, E_{0} \in \overline{\mathbb{R}}_{+}^{q}$, and $\mathscr{E}_{\mathrm{r}}\left(E_{0}\right)<\infty, E_{0} \in \overline{\mathbb{R}}_{+}^{q}$, respectively. Next, let $E_{0} \in \overline{\mathbb{R}}_{+}^{q}$ and let $S(\cdot) \in \mathcal{U}$ be such that $E\left(k_{\mathrm{i}}\right)=E\left(k_{\mathrm{f}}\right)=0$ and $E\left(k_{0}\right)=$ $E_{0}$, where $k_{\mathrm{i}} \leq k_{0} \leq k_{\mathrm{f}}$. In this case, it follows from (3.30) that

$$
\sum_{k=k_{\mathrm{i}}}^{k_{\mathrm{f}}-1} \sum_{i=1}^{q} E_{i}(k+1)\left[S_{i}(k)-\sigma_{i i}(E(k))\right] \geq 0,
$$


or, equivalently,

$$
\sum_{k=k_{\mathrm{i}}}^{k_{0}-1} \sum_{i=1}^{q} E_{i}(k+1)\left[S_{i}(k)-\sigma_{i i}(E(k))\right] \geq-\sum_{k=k_{0}}^{k_{\mathrm{f}}-1} \sum_{i=1}^{q} E_{i}(k+1)\left[S_{i}(k)-\sigma_{i i}(E(k))\right] .
$$

Now, taking the infimum on both sides of (3.37) over all $S(\cdot) \in U_{\mathrm{r}}$ and $k_{\mathrm{i}}+1 \leq k_{0}$ yields

$$
\begin{aligned}
\mathscr{E}_{\mathrm{r}}\left(E_{0}\right) & =\inf _{S(\cdot) \in \mathcal{U}_{\mathrm{r}}, k_{\mathrm{i}}+1 \leq k_{0}} \sum_{k=k_{\mathrm{i}}}^{k_{0}-1} \sum_{i=1}^{q} E_{i}(k+1)\left[S_{i}(k)-\sigma_{i i}(E(k))\right] \\
& \geq-\sum_{k=k_{0}}^{k_{\mathrm{f}}-1} \sum_{i=1}^{q} E_{i}(k+1)\left[S_{i}(k)-\sigma_{i i}(E(k))\right] .
\end{aligned}
$$

Next, taking the supremum on both sides of (3.38) over all $S(\cdot) \in \mathcal{U}_{\mathrm{c}}$ and $k_{\mathrm{f}} \geq k_{0}$, we obtain $\mathscr{E}_{\mathrm{r}}\left(E_{0}\right) \geq \mathscr{E}_{\mathrm{a}}\left(E_{0}\right), E_{0} \in \overline{\mathbb{R}}_{+}^{q}$, which implies that $-\infty<\mathscr{E}_{\mathrm{a}}\left(E_{0}\right) \leq \mathscr{E}_{\mathrm{r}}\left(E_{0}\right)<\infty, E_{0} \in$ $\overline{\mathbb{R}}_{+}^{q}$. Hence, the functions $\mathscr{E}_{\mathrm{a}}(\cdot)$ and $\mathscr{E}_{\mathrm{r}}(\cdot)$ are well defined.

Next, it follows from the definition of $\mathscr{E}_{\mathrm{a}}(\cdot)$ that, for any $K \geq k_{1}$ and $S(\cdot) \in \mathcal{U}_{\mathrm{c}}$ such that $E\left(k_{1}\right) \in \overline{\mathbb{R}}_{+}^{q}$ and $E(K)=0$,

$$
\begin{aligned}
-\mathscr{E}_{\mathrm{a}}\left(E\left(k_{1}\right)\right) \leq & \sum_{k=k_{1}}^{k_{2}-1} \sum_{i=1}^{q} E_{i}(k+1)\left[S_{i}(k)-\sigma_{i i}(E(k))\right] \\
& +\sum_{k=k_{2}}^{K-1} \sum_{i=1}^{q} E_{i}(k+1)\left[S_{i}(k)-\sigma_{i i}(E(k))\right], \quad k_{1} \leq k_{2} \leq K,
\end{aligned}
$$

and hence

$$
\begin{aligned}
-\mathscr{E}_{\mathrm{a}}\left(E\left(k_{1}\right)\right) \leq & \sum_{k=k_{1}}^{k_{2}-1} \sum_{i=1}^{q} E_{i}(k+1)\left[S_{i}(k)-\sigma_{i i}(E(k))\right] \\
& +\inf _{S(\cdot) \in \mathscr{U}_{\mathrm{c}}, K \geq k_{2}} \sum_{k=k_{2}}^{K-1} \sum_{i=1}^{q} E_{i}(k+1)\left[S_{i}(k)-\sigma_{i i}(E(k))\right] \\
= & \sum_{k=k_{1}}^{k_{2}-1} \sum_{i=1}^{q} E_{i}(k+1)\left[S_{i}(k)-\sigma_{i i}(E(k))\right]-\mathscr{E}_{\mathrm{a}}\left(E\left(k_{2}\right)\right),
\end{aligned}
$$

which implies that $\mathscr{E}_{\mathrm{a}}(E), E \in \overline{\mathbb{R}}_{+}^{q}$, satisfies (3.32). Thus, $\mathscr{E}_{\mathrm{a}}(E), E \in \overline{\mathbb{R}}_{+}^{q}$, is a possible ectropy function for the system $\mathscr{G}$. Note that with $E\left(k_{0}\right)=E(K)=0$, it follows from (3.30) that the infimum in (3.33) is taken over the set of nonnegative values with one of the values being zero for $S(k) \equiv 0$. Thus, $\mathscr{E}_{\mathrm{a}}(0)=0$. Similarly, it can be shown that $\mathscr{E}_{\mathrm{r}}(E)$, $E \in \overline{\mathbb{R}}_{+}^{q}$, given by (3.34) satisfies (3.32), and hence is a possible ectropy function for the system $\mathscr{G}$ with $\mathscr{E}_{\mathrm{r}}(0)=0$. 
292 Thermodynamic modeling for discrete-time systems

Next, suppose that there exists an ectropy function $\mathscr{E}: \overline{\mathbb{R}}_{+}^{q} \rightarrow \mathbb{R}$ for $\varphi$ and let $E\left(k_{2}\right)=0$ in (3.32). Then it follows from (3.32) that

$$
\mathscr{E}\left(E\left(k_{1}\right)\right)-\mathscr{E}(0) \geq-\sum_{k=k_{1}}^{k_{2}-1} \sum_{i=1}^{q} E_{i}(k+1)\left[S_{i}(k)-\sigma_{i i}(E(k))\right]
$$

for all $k_{2} \geq k_{1}$ and $S(\cdot) \in U_{\mathrm{c}}$, which implies that

$$
\begin{aligned}
\mathscr{E}\left(E\left(k_{1}\right)\right)-\mathscr{E}(0) & \geq \sup _{S(\cdot) \in \mathcal{U}_{c}, k_{2} \geq k_{1}}\left[-\sum_{k=k_{1}}^{k_{2}-1} \sum_{i=1}^{q} E_{i}(k+1)\left[S_{i}(k)-\sigma_{i i}(E(k))\right]\right] \\
& =-\inf _{S(\cdot) \in \mathscr{U}_{c}, k_{2} \geq k_{1}} \sum_{k=k_{1}}^{k_{2}-1} \sum_{i=1}^{q} E_{i}(k+1)\left[S_{i}(k)-\sigma_{i i}(E(k))\right] \\
& =\mathscr{E}_{\mathrm{a}}\left(E\left(k_{1}\right)\right) .
\end{aligned}
$$

Since $E\left(k_{1}\right)$ is arbitrary, it follows that $\mathscr{E}(E)-\mathscr{E}(0) \geq \mathscr{E}_{\mathrm{a}}(E), E \in \overline{\mathbb{R}}_{+}^{q}$. Alternatively, let $E\left(k_{1}\right)=0$ in (3.32). Then it follows from (3.32) that

$$
\mathscr{E}\left(E\left(k_{2}\right)\right)-\mathscr{E}(0) \leq \sum_{k=k_{1}}^{k_{2}-1} \sum_{i=1}^{q} E_{i}(k+1)\left[S_{i}(k)-\sigma_{i i}(E(k))\right]
$$

for all $k_{1}+1 \leq k_{2}$ and $S(\cdot) \in \mathcal{U}_{\mathrm{r}}$. Hence,

$$
\begin{aligned}
\mathscr{E}\left(E\left(k_{2}\right)\right)-\mathscr{E}(0) & \leq \inf _{S(\cdot) \in \mathscr{U}_{\mathrm{r}}, k_{1}+1 \leq k_{2}} \sum_{k=k_{1}}^{k_{2}-1} \sum_{i=1}^{q} E_{i}(k+1)\left[S_{i}(k)-\sigma_{i i}(E(k))\right] \\
& =\mathscr{E}_{\mathrm{r}}\left(E\left(k_{2}\right)\right),
\end{aligned}
$$

which, since $E\left(k_{2}\right)$ is arbitrary, implies that $\mathscr{E}_{\mathrm{r}}(E) \geq \mathscr{E}(E)-\mathscr{E}(0), E \in \overline{\mathbb{R}}_{+}^{q}$. Thus, all ectropy functions for $\mathscr{G}$ satisfy (3.35).

The next proposition gives a closed-form expression for the ectropy of $\mathscr{G}$.

Proposition 3.12. Consider the discrete-time large-scale dynamical system $\varphi$ with energy balance equation (3.2) and assume that Axioms 2 and 3 hold. Then the function $\mathscr{E}: \overline{\mathbb{R}}_{+}^{q} \rightarrow \mathbb{R}$ given by

$$
\mathscr{E}(E)=\frac{1}{2} E^{\mathrm{T}} E, \quad E \in \overline{\mathbb{R}}_{+}^{q},
$$

is an ectropy function of $\mathscr{G}$. 
Proof. Since $E(k) \geq \geq 0, k \geq k_{0}$, and $\phi_{i j}(E)=-\phi_{j i}(E), E \in \overline{\mathbb{R}}_{+}^{q}, i \neq j, i, j=1, \ldots, q$, it follows that

$$
\begin{aligned}
\Delta \mathscr{E}(E(k))= & \frac{1}{2} E^{\mathrm{T}}(k+1) E(k+1)-\frac{1}{2} E^{\mathrm{T}}(k) E(k) \\
= & \sum_{i=1}^{q} E_{i}(k+1)\left[S_{i}(k)-\sigma_{i i}(E(k))\right] \\
& -\frac{1}{2} \sum_{i=1}^{q}\left[\sum_{j=1, j \neq i}^{q} \phi_{i j}(E(k))+S_{i}(k)-\sigma_{i i}(E(k))\right]^{2} \\
& +\sum_{i=1}^{q} \sum_{j=1, j \neq i}^{q} E_{i}(k+1) \phi_{i j}(E(k)) \\
= & \sum_{i=1}^{q} E_{i}(k+1)\left[S_{i}(k)-\sigma_{i i}(E(k))\right] \\
& -\frac{1}{2} \sum_{i=1}^{q}\left[\sum_{j=1, j \neq i}^{q} \phi_{i j}(E(k))+S_{i}(k)-\sigma_{i i}(E(k))\right]^{2} \\
& +\sum_{i=1}^{q-1} \sum_{j=i+1}^{q}\left(E_{i}(k+1)-E_{j}(k+1)\right) \phi_{i j}(E(k)) \\
\leq & \sum_{i=1}^{q} E_{i}(k+1)\left[S_{i}(k)-\sigma_{i i}(E(k))\right], \quad k \geq k_{0} .
\end{aligned}
$$

Now, summing (3.46) over $\left\{k_{1}, \ldots, k_{2}-1\right\}$ yields (3.32).

Remark 3.13. Note that it follows from the last equality in (3.46) that the ectropy function given by (3.45) satisfies (3.32) as an equality for an equilibrium process and as a strict inequality for a nonequilibrium process.

It follows from (3.45) that ectropy is a measure of the extent to which the system energy deviates from a homogeneous state. Thus, ectropy is the dual of entropy and is a measure of the tendency of the discrete-time large-scale dynamical system $\mathscr{G}$ to do useful work and grow more organized.

3.4. Semistability of thermodynamic models. Inequality (3.15) is analogous to Clausius' inequality for equilibrium and nonequilibrium thermodynamics as applied to discrete-time large-scale dynamical systems; while inequality (3.32) is an anti-Clausius' inequality. Moreover, for the ectropy function defined by (3.45), inequality (3.46) shows that a thermodynamically consistent discrete-time large-scale dynamical system is dissipative [23] with respect to the supply rate $E^{\mathrm{T}} S$ and with storage function corresponding to the system ectropy $\mathscr{E}(E)$. For the entropy function given by $(3.28)$, note that $\mathscr{S}(0)=0$, or, 
equivalently, $\lim _{E \rightarrow 0} \mathscr{S}(E)=0$, which is consistent with the third law of thermodynamics (Nernst's theorem) which states that the entropy of every system at absolute zero can always be taken to be equal to zero.

For the isolated discrete-time large-scale dynamical system $\mathscr{G},(3.15)$ yields the fundamental inequality

$$
\mathscr{S}\left(E\left(k_{2}\right)\right) \geq \mathscr{S}\left(E\left(k_{1}\right)\right), \quad k_{2} \geq k_{1} .
$$

Inequality (3.47) implies that, for any dynamical change in an isolated (i.e., $S(k) \equiv 0$ and $d(E(k)) \equiv 0)$ discrete-time large-scale system, the entropy of the final state can never be less than the entropy of the initial state. It is important to stress that this result holds for an isolated dynamical system. It is however possible with energy supplied from an external dynamical system (e.g., a controller) to reduce the entropy of the discrete-time large-scale dynamical system. The entropy of both systems taken together however cannot decrease. The above observations imply that when an isolated discrete-time large-scale dynamical system with thermodynamically consistent energy flow characteristics (i.e., Axioms 1, 2, and 3 hold) is at a state of maximum entropy consistent with its energy, it cannot be subject to any further dynamical change since any such change would result in a decrease of entropy. This of course implies that the state of maximum entropy is the stable state of an isolated system and this state has to be semistable.

Analogously, it follows from (3.32) that for an isolated discrete-time large-scale dynamical system $\mathscr{G}$, the fundamental inequality

$$
\mathscr{E}\left(E\left(k_{2}\right)\right) \leq \mathscr{E}\left(E\left(k_{1}\right)\right), \quad k_{2} \geq k_{1}
$$

is satisfied, which implies that the ectropy of the final state of $\varphi$ is always less than or equal to the ectropy of the initial state of $\mathscr{G}$. Hence, for the isolated large-scale dynamical system $\mathscr{G}$, the entropy increases if and only if the ectropy decreases. Thus, the state of minimum ectropy is the stable state of an isolated system and this equilibrium state has to be semistable. The next theorem concretizes the above observations.

Theorem 3.14. Consider the discrete-time large-scale dynamical system $G$ with energy balance equation (3.2) with $S(k) \equiv 0$ and $d(E) \equiv 0$, and assume that Axioms 1, 2, and 3 hold. Then for every $\alpha \geq 0$, $\alpha \mathbf{e}$ is a Lyapunov equilibrium state of (3.2). Furthermore, $E(k) \rightarrow(1 / q) \mathbf{e e}^{\mathrm{T}} E\left(k_{0}\right)$ as $k \rightarrow \infty$ and $(1 / q) \mathbf{e e}^{\mathrm{T}} E\left(k_{0}\right)$ is a semistable equilibrium state. Finally, if for some $m \in\{1, \ldots, q\}, \sigma_{m m}(E) \geq 0, E \in \overline{\mathbb{R}}_{+}^{q}$, and $\sigma_{m m}(E)=0$ if and only if $E_{m}=0$, then the zero solution $E(k) \equiv 0$ to (3.2) is a globally asymptotically stable equilibrium state of (3.2).

Proof. It follows from Axiom 1 that $\alpha \mathbf{e} \in \overline{\mathbb{R}}_{+}^{q}, \alpha \geq 0$, is an equilibrium state for (3.2). To show Lyapunov stability of the equilibrium state $\alpha \mathbf{e}$, consider the system shifted ectropy $\mathscr{E}_{s}(E)=(1 / 2)(E-\alpha \mathbf{e})^{\mathrm{T}}(E-\alpha \mathbf{e})$ as a Lyapunov function candidate. Now, since $\phi_{i j}(E)=$ $-\phi_{j i}(E), E \in \overline{\mathbb{R}}_{+}^{q}, i \neq j, i, j=1, \ldots, q$, and $\mathrm{e}^{\mathrm{T}} E(k+1)=\mathrm{e}^{\mathrm{T}} E(k), k \geq k_{0}$, it follows from 
Axioms 2 and 3 that

$$
\begin{aligned}
\Delta \mathscr{E}_{s}(E(k)) & =\frac{1}{2}(E(k+1)-\alpha \mathbf{e})^{\mathrm{T}}(E(k+1)-\alpha \mathbf{e})-\frac{1}{2}(E(k)-\alpha \mathbf{e})^{\mathrm{T}}(E(k)-\alpha \mathbf{e}) \\
& =\sum_{i=1}^{q} \sum_{j=1, j \neq i}^{q} E_{i}(k+1) \phi_{i j}(E(k))-\frac{1}{2} \sum_{i=1}^{q}\left[\sum_{j=1, j \neq i}^{q} \phi_{i j}(E(k))\right]^{2} \\
& =\sum_{i=1}^{q-1} \sum_{j=i+1}^{q}\left(E_{i}(k+1)-E_{j}(k+1)\right) \phi_{i j}(E(k))-\frac{1}{2} \sum_{i=1}^{q}\left[\sum_{j=1, j \neq i}^{q} \phi_{i j}(E(k))\right]^{2} \\
& \leq 0, \quad E(k) \in \overline{\mathbb{R}}_{+}^{q}, k \geq k_{0},
\end{aligned}
$$

which establishes Lyapunov stability of the equilibrium state $\alpha \mathbf{e}$.

To show that $\alpha \mathbf{e}$ is semistable, note that

$$
\begin{aligned}
\Delta \mathscr{E}_{s}(E(k)) & =\sum_{i=1}^{q} \sum_{j=1, j \neq i}^{q} E_{i}(k) \phi_{i j}(E(k))+\frac{1}{2} \sum_{i=1}^{q}\left[\sum_{j=1, j \neq i}^{q} \phi_{i j}(E(k))\right]^{2} \\
& \geq \sum_{i=1}^{q-1} \sum_{j=i+1}^{q}\left(E_{i}(k)-E_{j}(k)\right) \phi_{i j}(E(k)) \\
& =\sum_{i=1}^{q-1} \sum_{j \in \mathscr{K}_{i}}\left(E_{i}(k)-E_{j}(k)\right) \phi_{i j}(E(k)), \quad E(k) \in \overline{\mathbb{R}}_{+}^{q}, k \geq k_{0},
\end{aligned}
$$

where $\mathcal{K}_{i} \triangleq \mathcal{N}_{i} \backslash \cup_{l=1}^{i-1}\{l\}$ and $\mathcal{N}_{i} \triangleq\left\{j \in\{1, \ldots, q\}: \phi_{i j}(E)=0\right.$ if and only if $\left.E_{i}=E_{j}\right\}, i=$ $1, \ldots, q$.

Next, we show that $\Delta \mathscr{E}_{s}(E)=0$ if and only if $\left(E_{i}-E_{j}\right) \phi_{i j}(E)=0, i=1, \ldots, q, j \in \mathscr{K}_{i}$. First, assume that $\left(E_{i}-E_{j}\right) \phi_{i j}(E)=0, i=1, \ldots, q, j \in \mathcal{K}_{i}$. Then it follows from (3.50) that $\Delta \mathscr{E}_{s}(E) \geq 0$. However, it follows from (3.49) that $\Delta \mathscr{E}_{s}(E) \leq 0$. Hence, $\Delta \mathscr{E}_{s}(E)=0$. Conversely, assume that $\Delta \mathscr{E}_{s}(E)=0$. In this case, it follows from $(3.49)$ that $\left(E_{i}(k+1)-\right.$ $\left.E_{j}(k+1)\right) \phi_{i j}(E(k))=0$ and $\sum_{j=1, j \neq i}^{q} \phi_{i j}(E(k))=0, k \geq k_{0}, i, j=1, \ldots, q, i \neq j$. Since

$$
\begin{aligned}
{\left[E_{i}(k+1)-E_{j}(k+1)\right] \phi_{i j}(E(k))=} & {\left[E_{i}(k)-E_{j}(k)\right] \phi_{i j}(E(k)) } \\
& +\left[\sum_{h=1, h \neq i}^{q} \phi_{i h}(E(k))-\sum_{l=1, l \neq j}^{q} \phi_{j l}(E(k))\right] \phi_{i j}(E(k)) \\
= & {\left[E_{i}(k)-E_{j}(k)\right] \phi_{i j}(E(k)), \quad k \geq k_{0}, i, j=1, \ldots, q, i \neq j, }
\end{aligned}
$$

it follows that $\left(E_{i}-E_{j}\right) \phi_{i j}(E)=0, i=1, \ldots, q, j \in \mathscr{K}_{i}$.

Let $\mathscr{R} \triangleq\left\{E \in \overline{\mathbb{R}}_{+}^{q}: \Delta \mathscr{E}_{s}(E)=0\right\}=\left\{E \in \overline{\mathbb{R}}_{+}^{q}:\left(E_{i}-E_{j}\right) \phi_{i j}(E)=0, i=1, \ldots, q, j \in \mathscr{K}_{i}\right\}$. Now, by Axiom 1, the directed graph associated with the connectivity matrix $\mathscr{C}$ for the discrete-time large-scale dynamical system $\mathscr{G}$ is strongly connected which implies that $\mathscr{R}=\left\{E \in \overline{\mathbb{R}}_{+}^{q}: E_{1}=\cdots=E_{q}\right\}$. Since the set $\mathscr{R}$ consists of the equilibrium states of (3.2), it follows that the largest invariant set $\mathcal{M}$ contained in $\mathscr{R}$ is given by $\mathcal{M}=\mathscr{R}$. Hence, it 
follows from LaSalle's invariant set theorem that for any initial condition $E\left(k_{0}\right) \in \overline{\mathbb{R}}_{+}^{q}$, $E(k) \rightarrow M$ as $k \rightarrow \infty$, and hence $\alpha \mathbf{e}$ is a semistable equilibrium state of (3.2). Next, note that since $\mathbf{e}^{\mathrm{T}} E(k)=\mathbf{e}^{\mathrm{T}} E\left(k_{0}\right)$ and $E(k) \rightarrow \mathcal{M}$ as $k \rightarrow \infty$, it follows that $E(k) \rightarrow(1 / q) \mathbf{e e}^{\mathrm{T}} E\left(k_{0}\right)$ as $k \rightarrow \infty$. Hence, with $\alpha=(1 / q) \mathbf{e}^{\mathrm{T}} E\left(k_{0}\right), \alpha \mathbf{e}=(1 / q) \mathbf{e e}^{\mathrm{T}} E\left(k_{0}\right)$ is a semistable equilibrium state of (3.2).

Finally, to show that in the case where for some $m \in\{1, \ldots, q\}, \sigma_{m m}(E) \geq 0, E \in \overline{\mathbb{R}}_{+}^{q}$, and $\sigma_{m m}(E)=0$ if and only if $E_{m}=0$, the zero solution $E(k) \equiv 0$ to (3.2) is globally asymptotically stable, consider the system ectropy $\mathscr{E}(E)=(1 / 2) E^{\mathrm{T}} E$ as a candidate Lyapunov function. Note that $\mathscr{E}(0)=0, \mathscr{E}(E)>0, E \in \overline{\mathbb{R}}_{+}^{q}, E \neq 0$, and $\mathscr{E}(E)$ is radially unbounded. Now, the Lyapunov difference is given by

$$
\begin{aligned}
\Delta \mathscr{E}(E(k))= & \frac{1}{2} E^{\mathrm{T}}(k+1) E(k+1)-\frac{1}{2} E^{\mathrm{T}}(k) E(k) \\
= & -E_{m}(k+1) \sigma_{m m}(E(k))-\frac{1}{2}\left[\sum_{j=1, j \neq m}^{q} \phi_{m j}(E(k))-\sigma_{m m}(E(k))\right]^{2} \\
& -\frac{1}{2} \sum_{i=1, i \neq m}^{q}\left[\sum_{j=1, j \neq i}^{q} \phi_{i j}(E(k))\right]^{2}+\sum_{i=1}^{q} \sum_{j=1, j \neq i}^{q} E_{i}(k+1) \phi_{i j}(E(k)) \\
= & -E_{m}(k+1) \sigma_{m m}(E(k))-\frac{1}{2}\left[\sum_{j=1, j \neq m}^{q} \phi_{m j}(E(k))-\sigma_{m m}(E(k))\right]^{2} \\
& -\frac{1}{2} \sum_{i=1, i \neq m}^{q}\left[\sum_{j=1, j \neq i}^{q} \phi_{i j}(E(k))\right]^{2}+\sum_{i=1}^{q-1} \sum_{j=i+1}^{q}\left(E_{i}(k+1)-E_{j}(k+1)\right) \phi_{i j}(E(k)) \\
\leq & 0, \quad E(k) \in \overline{\mathbb{R}}_{+}^{q}, k \geq k_{0},
\end{aligned}
$$

which shows that the zero solution $E(k) \equiv 0$ to (3.2) is Lyapunov stable.

To show global asymptotic stability of the zero equilibrium state, note that

$$
\begin{aligned}
\Delta \mathscr{E}(E(k))= & \sum_{i=1}^{q-1} \sum_{j=i+1}^{q}\left(E_{i}(k)-E_{j}(k)\right) \phi_{i j}(E(k))+\frac{1}{2} \sum_{i=1, i \neq m}^{q}\left[\sum_{j=1, j \neq i}^{q} \phi_{i j}(E(k))\right]^{2} \\
& -E_{m}(k) \sigma_{m m}(E(k))+\frac{1}{2}\left[\sum_{j=1, j \neq m}^{q} \phi_{m j}(E(k))-\sigma_{m m}(E(k))\right]^{2} \\
\geq & \sum_{i=1}^{q-1} \sum_{j \in \mathscr{K}_{i}}\left(E_{i}(k)-E_{j}(k)\right) \phi_{i j}(E(k))-E_{m}(k) \sigma_{m m}(E(k)), \quad E(k) \in \overline{\mathbb{R}}_{+}^{q}, k \geq k_{0} .
\end{aligned}
$$

Next, we show that $\Delta \mathscr{E}(E)=0$ if and only if $\left(E_{i}-E_{j}\right) \phi_{i j}(E)=0$ and $\sigma_{m m}(E)=0, i=$ $1, \ldots, q, j \in \mathcal{K}_{i}, m \in\{1, \ldots, q\}$. First, assume that $\left(E_{i}-E_{j}\right) \phi_{i j}(E)=0$ and $\sigma_{m m}(E)=0$, $i=1, \ldots, q, j \in \mathcal{K}_{i}, m \in\{1, \ldots, q\}$. Then it follows from (3.53) that $\Delta \mathscr{E}(E) \geq 0$. However, 
it follows from $(3.52)$ that $\Delta \mathscr{E}(E) \leq 0$. Thus, $\Delta \mathscr{E}(E)=0$. Conversely, assume that $\Delta \mathscr{E}(E)=$ 0 . Then it follows from $(3.52)$ that $\left(E_{i}(k+1)-E_{j}(k+1)\right) \phi_{i j}(E(k))=0, i, j=1, \ldots, q, i \neq j$, $\sum_{j=1, j \neq i}^{q} \phi_{i j}(E(k))=0, i=1, \ldots, q, i \neq m, k \geq k_{0}$, and $\sigma_{m m}(E)=0, m \in\{1, \ldots, q\}$. Note that in this case, it follows that $\sigma_{m m}(E)=\sum_{j=1, j \neq m}^{q} \phi_{m j}(E)=0$, and hence

$\left[E_{i}(k+1)-E_{j}(k+1)\right] \phi_{i j}(E(k))=\left[E_{i}(k)-E_{j}(k)\right] \phi_{i j}(E(k)), \quad k \geq k_{0}, i, j=1, \ldots, q, i \neq j$,

which implies that $\left(E_{i}-E_{j}\right) \phi_{i j}(E)=0, i=1, \ldots, q, j \in \mathcal{K}_{i}$. Hence, $\left(E_{i}-E_{j}\right) \phi_{i j}(E)=0$ and $\sigma_{m m}(E)=0, i=1, \ldots, q, j \in \mathcal{K}_{i}, m \in\{1, \ldots, q\}$ if and only if $\Delta \mathscr{E}(E)=0$.

Let $\mathscr{R} \triangleq\left\{E \in \overline{\mathbb{R}}_{+}^{q}: \Delta \mathscr{E}(E)=0\right\}=\left\{E \in \overline{\mathbb{R}}_{+}^{q}: \sigma_{m m}(E)=0, m \in\{1, \ldots, q\}\right\} \cap\left\{E \in \overline{\mathbb{R}}_{+}^{q}\right.$ : $\left.\left(E_{i}-E_{j}\right) \phi_{i j}(E)=0, i=1, \ldots, q, j \in \mathscr{K}_{i}\right\}$. Now, since Axiom 1 holds and $\sigma_{m m}(E)=0$ if and only if $E_{m}=0$, it follows that $\mathscr{R}=\left\{E \in \overline{\mathbb{R}}_{+}^{q}: E_{m}=0, m \in\{1, \ldots, q\}\right\} \cap\left\{E \in \overline{\mathbb{R}}_{+}^{q}\right.$ : $\left.E_{1}=E_{2}=\cdots=E_{q}\right\}=\{0\}$ and the largest invariant set $M$ contained in $\mathscr{R}$ is given by $M=$ $\{0\}$. Hence, it follows from LaSalle's invariant set theorem that for any initial condition $E\left(k_{0}\right) \in \overline{\mathbb{R}}_{+}^{q}, E(k) \rightarrow M=\{0\}$ as $k \rightarrow \infty$, which proves global asymptotic stability of the zero equilibrium state of (3.2).

Remark 3.15. The assumption $\sigma_{m m}(E) \geq 0, E \in \overline{\mathbb{R}}_{+}^{q}$, and $\sigma_{m m}(E)=0$ if and only if $E_{m}=0$ for some $m \in\{1, \ldots, q\}$ implies that if the $m$ th subsystem possesses no energy, then this subsystem cannot dissipate energy to the environment. Conversely, if the $m$ th subsystem does not dissipate energy to the environment, then this subsystem has no energy.

Remark 3.16. It is important to note that Axiom 3 involving monotonicity of solutions is explicitly used to prove semistability for discrete-time compartmental dynamical systems. However, Axiom 3 is a sufficient condition and not necessary for guaranteeing semistability. Replacing the monotonicity condition with $\sum_{i=1, j=1, i \neq j}^{q} \alpha_{i j}(E) f_{i j}(E) \geq 0$, where

$$
\begin{gathered}
\alpha_{i j}(E) \triangleq \begin{cases}\frac{\phi_{i j}(E)}{E_{j}-E_{i}}, & E_{i} \neq E_{j}, \\
0, & E_{i}=E_{j},\end{cases} \\
f_{i j}(E) \triangleq\left[E_{i}(k)-E_{j}(k)\right]\left[E_{i}(k+1)-E_{j}(k+1)\right],
\end{gathered}
$$

provides a weaker sufficient condition for guaranteeing semistability. However, in this case, to ensure that the entropy of $\mathscr{G}$ is monotonically increasing, we additionally require that $\sum_{i=1, j=1, i \neq j}^{q} \beta_{i j}(E) f_{i j}(E) \geq 0$, where

$$
\beta_{i j}(E) \triangleq \begin{cases}\frac{1}{\left(c+E_{i}(k+1)\right)\left(c+E_{j}(k+1)\right)} \cdot \frac{\phi_{i j}(E(k))}{E_{j}(k)-E_{i}(k)}, & E_{i} \neq E_{j}, \\ 0, & E_{i}=E_{j} .\end{cases}
$$




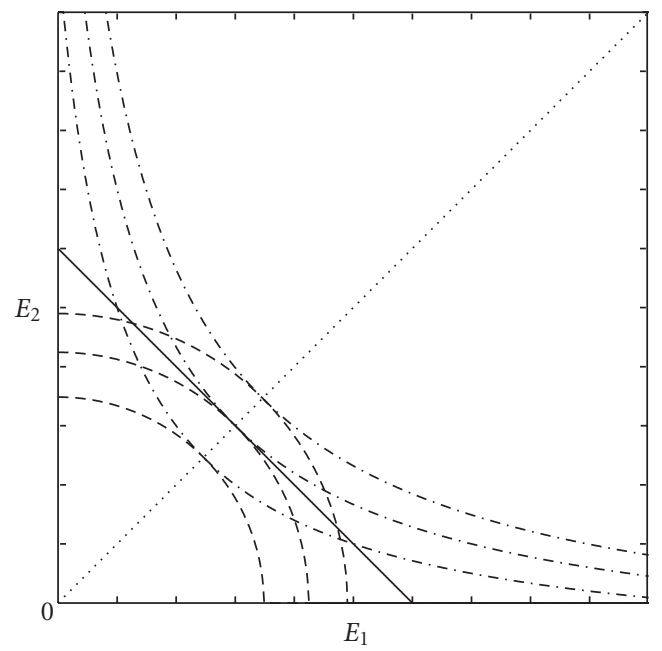

Figure 3.2. Thermodynamic equilibria $(\cdots)$, constant energy surfaces $(-)$, constant ectropy surfaces $(---)$, and constant entropy surfaces $(-\cdot-\cdot-)$.

Thus, a weaker condition for Axiom 3, which combines $\sum_{i=1, j=1, i \neq j}^{q} \alpha_{i j}(E) f_{i j}(E) \geq 0$ and $\sum_{i=1, j=1, i \neq j}^{q} \beta_{i j}(E) f_{i j}(E) \geq 0$, is $\sum_{i=1, j=1, i \neq j}^{q} \gamma_{i j}(E) f_{i j}(E) \geq 0$, where $\gamma_{i j}(E) \triangleq \alpha_{i j}(E)+\beta_{i j}(E)$ $-\operatorname{sgn}\left(f_{i j}(E)\right)\left|\alpha_{i j}(E)-\beta_{i j}(E)\right|$ and $\operatorname{sgn}\left(f_{i j}(E)\right) \triangleq\left|f_{i j}(E)\right| / f_{i j}(E)$.

In Theorem 3.14, we used the shifted ectropy function to show that for the isolated (i.e., $S(k) \equiv 0$ and $d(E) \equiv 0)$ discrete-time large-scale dynamical system $\mathscr{G}$ with Axioms 1 , 2, and $3, E(k) \rightarrow(1 / q) \mathbf{e e}^{\mathrm{T}} E\left(k_{0}\right)$ as $k \rightarrow \infty$ and $(1 / q) \mathbf{e e}^{\mathrm{T}} E\left(k_{0}\right)$ is a semistable equilibrium state. This result can also be arrived at using the system entropy for the isolated discretetime large-scale dynamical system $\mathscr{G}$ with Axioms 1,2 , and 3 . To see this note that since $\mathbf{e}^{\mathrm{T}} w(E)=\mathbf{e}^{\mathrm{T}} E, E \in \overline{\mathbb{R}}_{+}^{q}$, it follows that $\mathbf{e}^{\mathrm{T}} \Delta E(k)=0, k \geq k_{0}$. Hence, $\mathbf{e}^{\mathrm{T}} E(k)=\mathbf{e}^{\mathrm{T}} E\left(k_{0}\right), k \geq$ $k_{0}$. Furthermore, since $E(k) \geq \geq 0, k \geq k_{0}$, it follows that $0 \leq \leq E(k) \leq \leq \mathbf{e e}^{\mathrm{T}} E\left(k_{0}\right), k \geq k_{0}$, which implies that all solutions to (3.2) are bounded. Next, since by (3.47) the entropy

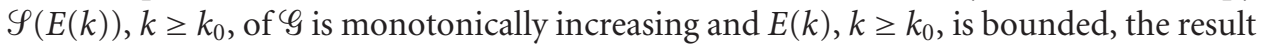
follows by using similar arguments as in Theorem 3.14 and using the fact that $x /(1+x) \leq$ $\log _{e}(1+x) \leq x$ for all $x>-1$.

3.5. Energy equipartition. Theorem 3.14 implies that the steady-state value of the en-

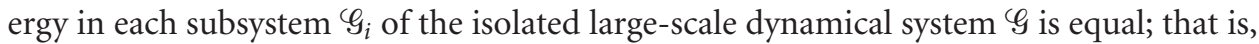
the steady-state energy of the isolated discrete-time large-scale dynamical system $\varphi$ given by $E_{\infty}=(1 / q) \mathbf{e e}^{\mathrm{T}} E\left(k_{0}\right)=\left[(1 / q) \sum_{i=1}^{q} E_{i}\left(k_{0}\right)\right] \mathbf{e}$ is uniformly distributed over all subsystems of $\mathscr{G}$. This phenomenon is known as equipartition of energy (the phenomenon of equipartition of energy is closely related to the notion of a monotemperaturic system discussed in $[6])[4,5,14,18,21]$ and is an emergent behavior in thermodynamic systems. The next proposition shows that among all possible energy distributions in the discrete-time large-scale dynamical system $\mathscr{G}$, energy equipartition corresponds to the minimum value of the system's ectropy and the maximum value of the system's entropy (see Figure 3.2). 
Proposition 3.17. Consider the discrete-time large-scale dynamical system $\varphi$ with energy balance equation (3.2), let $\mathscr{E}: \overline{\mathbb{R}}_{+}^{q} \rightarrow \mathbb{R}$ and $\mathscr{Y}: \overline{\mathbb{R}}_{+}^{q} \rightarrow \mathbb{R}$ denote the ectropy and entropy of $\mathscr{G}$ given by (3.45) and (3.28), respectively, and define $\mathscr{D}_{\mathrm{c}} \triangleq\left\{E \in \overline{\mathbb{R}}_{+}^{q}: \mathrm{e}^{\mathrm{T}} E=\beta\right\}$, where $\beta \geq 0$. Then,

$$
\underset{E \in \mathscr{D}_{\mathfrak{c}}}{\arg \min }(\mathscr{E}(E))=\underset{E \in \mathscr{D}_{\mathfrak{c}}}{\arg \max }(\mathscr{Y}(E))=E^{*}=\frac{\beta}{q} \mathbf{e} .
$$

Furthermore, $\mathscr{E}_{\min } \triangleq \mathscr{E}\left(E^{*}\right)=(1 / 2)\left(\beta^{2} / q\right)$ and $\mathscr{S}_{\max } \triangleq \mathscr{S}\left(E^{*}\right)=q \log _{e}(c+\beta / q)-q \log _{e} c$.

Proof. The existence and uniqueness of $E^{*}$ follow from the fact that $\mathscr{E}(E)$ and $-\mathscr{S}(E)$ are strictly convex continuous functions on the compact set $\mathscr{D}_{c}$. To minimize $\mathscr{E}(E)=$ $(1 / 2) E^{\mathrm{T}} E, E \in \overline{\mathbb{R}}_{+}^{q}$, subject to $E \in \mathscr{D}_{\mathrm{c}}$, form the Lagrangian $\mathscr{L}(E, \lambda)=(1 / 2) E^{\mathrm{T}} E+\lambda\left(\mathbf{e}^{\mathrm{T}} E-\right.$ $\beta$ ), where $\lambda \in \mathbb{R}$ is the Lagrange multiplier. If $E^{*}$ solves this minimization problem, then

$$
0=\left.\frac{\partial \mathscr{L}}{\partial E}\right|_{E=E^{*}}=E^{* \mathrm{~T}}+\lambda \mathbf{e}^{\mathrm{T}}
$$

and hence $E^{*}=-\lambda \mathbf{e}$. Now, it follows from $\mathbf{e}^{\mathrm{T}} E=\beta$ that $\lambda=-(\beta / q)$ which implies that $E^{*}=(\beta / q) \mathbf{e} \in \overline{\mathbb{R}}_{+}^{q}$. The fact that $E^{*}$ minimizes the ectropy on the compact set $\mathscr{D}_{\mathrm{c}}$ can be shown by computing the Hessian of the ectropy for the constrained parameter optimization problem and showing that the Hessian is positive definite at $E^{*} . \mathscr{E}_{\min }=(1 / 2)\left(\beta^{2} / q\right)$ is now immediate.

Analogously, to maximize $\mathscr{S}(E)=\mathbf{e}^{\mathrm{T}} \log _{e}(c \mathbf{e}+E)-q \log _{e} c$ on the compact set $\mathscr{D}_{\mathrm{c}}$, form the Lagrangian $\mathscr{L}(E, \lambda) \triangleq \sum_{i=1}^{q} \log _{e}\left(c+E_{i}\right)+\lambda\left(\mathbf{e}^{\mathrm{T}} E-\beta\right)$, where $\lambda \in \mathbb{R}$ is a Lagrange multiplier. If $E^{*}$ solves this maximization problem, then

$$
0=\left.\frac{\partial \mathscr{L}}{\partial E}\right|_{E=E^{*}}=\left[\frac{1}{c+E_{1}^{*}}+\lambda, \ldots, \frac{1}{c+E_{q}^{*}}+\lambda\right]
$$

Thus, $\lambda=-\left(1 /\left(c+E_{i}^{*}\right)\right), i=1, \ldots, q$. If $\lambda=0$, then the only value of $E^{*}$ that satisfies (3.59) is $E^{*}=\infty$, which does not satisfy the constraint equation $\mathrm{e}^{\mathrm{T}} E=\beta$ for finite $\beta \geq 0$. Hence, $\lambda \neq 0$ and $E_{i}^{*}=-(1 / \lambda+c), i=1, \ldots, q$, which implies that $E^{*}=-(1 / \lambda+c)$ e. Now, it follows from $\mathbf{e}^{\mathrm{T}} E=\beta$ that $-(1 / \lambda+c)=\beta / q$, and hence $E^{*}=(\beta / q) \mathbf{e} \in \overline{\mathbb{R}}_{+}^{q}$. The fact that $E^{*}$ maximizes the entropy on the compact set $\mathscr{D}_{\mathrm{c}}$ can be shown by computing the Hessian and showing that it is negative definite at $E^{*} . \mathscr{Y}_{\max }=q \log _{e}(c+\beta / q)-q \log _{e} c$ is now immediate.

It follows from (3.47), (3.48), and Proposition 3.17 that conservation of energy necessarily implies nonconservation of ectropy and entropy. Hence, in an isolated discrete-time large-scale dynamical system $\mathscr{G}$, all the energy, though always conserved, will eventually be degraded (diluted) to the point where it cannot produce any useful work. Hence, all motion would cease and the dynamical system would be fated to a state of eternal rest 
(semistability), wherein all subsystems will possess identical energies (energy equipartition). Ectropy would be a minimum and entropy would be a maximum giving rise to a state of absolute disorder. This is precisely what is known in theoretical physics as the heat death of the universe [13].

3.6. Entropy increase and the second law of thermodynamics. In the preceding discussion, it was assumed that our discrete-time large-scale nonlinear dynamical system model is such that energy is exchanged from more energetic subsystems to less energetic subsystems; that is, heat (energy) flows in the direction of lower temperatures. Although this universal phenomenon can be predicted with virtual certainty, it follows as a manifestation of entropy and ectropy nonconservation for the case of two subsystems. To see this, consider the isolated (i.e., $S(k) \equiv 0$ and $d(E) \equiv 0$ ) discrete-time large-scale dynamical system $\mathscr{G}$ with energy balance equation (3.2) and assume that the system entropy is monotonically increasing, and hence $\Delta \mathscr{S}(E(k)) \geq 0, k \geq k_{0}$. Now, since

$$
\begin{aligned}
0 & \leq \Delta \mathscr{Y}(E(k)) \\
& =\sum_{i=1}^{q} \log _{e}\left[1+\frac{\Delta E_{i}(k)}{c+E_{i}(k)}\right] \\
& \leq \sum_{i=1}^{q} \frac{\Delta E_{i}(k)}{c+E_{i}(k)} \\
& =\sum_{i=1}^{q} \sum_{j=1, j \neq i}^{q} \frac{\phi_{i j}(E(k))}{c+E_{i}(k)} \\
& =\sum_{i=1}^{q-1} \sum_{j=i+1}^{q}\left[\frac{\phi_{i j}(E(k))}{c+E_{i}(k)}-\frac{\phi_{i j}(E(k))}{c+E_{j}(k)}\right] \\
& =\sum_{i=1}^{q-1} \sum_{j=i+1}^{q} \frac{\phi_{i j}(E(k))\left(E_{j}(k)-E_{i}(k)\right)}{\left(c+E_{i}(k)\right)\left(c+E_{j}(k)\right)}, \quad k \geq k_{0},
\end{aligned}
$$

it follows that for $q=2,\left(E_{1}-E_{2}\right) \phi_{12}(E) \leq 0, E \in \overline{\mathbb{R}}_{+}^{2}$, which implies that energy (heat) flows naturally from a more energetic subsystem (hot object) to a less energetic subsystem (cooler object). The universality of this emergent behavior thus follows from the fact that entropy (resp., ectropy) transfer, accompanying energy transfer, always increases (resp., decreases).

In the case where we have multiple subsystems, it is clear from (3.60) that entropy and ectropy nonconservation does not necessarily imply Axiom 2. However, if we invoke the additional condition (Axiom 4 ) that if for any pair of connected subsystems $\mathscr{G}_{k}$ and $\mathscr{G}_{l}, k \neq l$, with energies $E_{k} \geq E_{l}$ (resp., $E_{k} \leq E_{l}$ ), and for any other pair of connected subsystems $\mathscr{G}_{m}$ and $\mathscr{G}_{n}, m \neq n$, with energies $E_{m} \geq E_{n}$ (resp., $E_{m} \leq E_{n}$ ), the inequality $\phi_{k l}(E) \phi_{m n}(E) \geq 0, E \in \overline{\mathbb{R}}_{+}^{q}$, holds, then nonconservation of entropy and ectropy in the isolated discrete-time large-scale dynamical system $\mathscr{G}$ implies Axiom 2 . The above inequality 
postulates that the direction of energy exchange for any pair of energy similar subsystems is consistent; that is, if for a given pair of connected subsystems at given different energy levels the energy flows in a certain direction, then for any other pair of connected subsystems with the same energy level, the energy flow direction is consistent with the original pair of subsystems. Note that this assumption does not specify the direction of energy flow between subsystems.

To see that $\Delta \mathscr{Y}(E(k)) \geq 0, k \geq k_{0}$, along with Axiom 4, implies Axiom 2, note that since (3.60) holds for all $k \geq k_{0}$ and $E\left(k_{0}\right) \in \overline{\mathbb{R}}_{+}^{q}$ is arbitrary, (3.60) implies that

$$
\sum_{i=1}^{q} \sum_{j \in \mathscr{K}_{i}} \frac{\phi_{i j}(E)\left(E_{j}-E_{i}\right)}{\left(c+E_{i}\right)\left(c+E_{j}\right)} \geq 0, \quad E \in \overline{\mathbb{R}}_{+}^{q} .
$$

Now, it follows from (3.61) that for any fixed system energy level $E \in \overline{\mathbb{R}}_{+}^{q}$, there exists at least one pair of connected subsystems $\mathscr{G}_{k}$ and $\mathscr{G}_{l}, k \neq l$, such that $\phi_{k l}(E)\left(E_{l}-E_{k}\right) \geq$ 0 . Thus, if $E_{k} \geq E_{l}$ (resp., $E_{k} \leq E_{l}$ ), then $\phi_{k l}(E) \leq 0$ (resp., $\phi_{k l}(E) \geq 0$ ). Furthermore, it follows from Axiom 4 that for any other pair of connected subsystems $\varphi_{m}$ and $\varphi_{n}, m \neq n$, with $E_{m} \geq E_{n}$ (resp., $E_{m} \leq E_{n}$ ), the inequality $\phi_{m n}(E) \leq 0$ (resp., $\phi_{m n}(E) \geq 0$ ) holds which implies that

$$
\phi_{m n}(E)\left(E_{n}-E_{m}\right) \geq 0, \quad m \neq n .
$$

Thus, it follows from (3.62) that energy (heat) flows naturally from more energetic subsystems (hot objects) to less energetic subsystems (cooler objects). Of course, since in the isolated discrete-time large-scale dynamical system $\mathscr{G}$, ectropy decreases if and only if entropy increases, the same result can be arrived at by considering the ectropy of $\mathscr{G}$. Since Axiom 2 holds, it follows from the conservation of energy and the fact that the discrete-time large-scale dynamical system $\mathscr{G}$ is strongly connected that nonconservation of entropy and ectropy necessarily implies energy equipartition.

Finally, we close this section by showing that our definition of entropy given by (3.28) satisfies the eight criteria established in [10] for the acceptance of an analytic expression for representing a system entropy function. In particular, note that for a dynamical system $\mathscr{G}$, the following hold. (i) $\mathscr{S}(E)$ is well defined for every state $E \in \overline{\mathbb{R}}_{+}^{q}$ as long as $c>0$. (ii) If $\mathscr{G}$ is isolated, then $\mathscr{S}(E(k))$ is a nondecreasing function of time. (iii) If $\mathscr{S}_{i}\left(E_{i}\right)=\log _{e}\left(c+E_{i}\right)-\log _{e} c$ is the entropy of the $i$ th subsystem of the system $\mathscr{G}$, then $\mathscr{S}(E)=\sum_{i=1}^{q} \mathscr{I}_{i}\left(E_{i}\right)=\mathbf{e}^{\mathrm{T}} \log _{e}(c \mathbf{e}+E)-q \log _{e} c$, and hence the system entropy $\mathscr{S}(E)$ is an additive quantity over all subsystems. (iv) For the system $\mathscr{G}, \mathscr{S}(E) \geq 0$ for all $E \in \overline{\mathbb{R}}_{+}^{q}$. (v) It follows from Proposition 3.17 that for a given value $\beta \geq 0$ of the total energy of the system $\mathscr{G}$, one and only one state; namely, $E^{*}=(\beta / q) \mathbf{e}$, corresponds to the largest value of $\mathscr{S}(E)$. (vi) It follows from (3.28) that for the system $\mathscr{G}$, graph of entropy versus energy is concave and smooth. (vii) For a composite discrete-time large-scale dynamical system $\mathscr{G}_{\mathrm{C}}$ of two dynamical systems $\mathscr{G}_{\mathrm{A}}$ and $\mathscr{G}_{\mathrm{B}}$ the expression for the composite entropy $\mathscr{S}_{\mathrm{C}}=\mathscr{Y}_{\mathrm{A}}+\mathscr{S}_{\mathrm{B}}$, where $\mathscr{S}_{\mathrm{A}}$ and $\mathscr{S}_{\mathrm{B}}$ are entropies of $\mathscr{G}_{\mathrm{A}}$ and $\mathscr{G}_{\mathrm{B}}$, respectively, is such that the expression for the equilibrium state where the composite maximum entropy is achieved is identical to those obtained for $\mathscr{G}_{\mathrm{A}}$ and $\mathscr{G}_{\mathrm{B}}$ individually. Specifically, if $q_{\mathrm{A}}$ and $q_{\mathrm{B}}$ denote 
the number of subsystems in $\mathscr{G}_{\mathrm{A}}$ and $\mathscr{G}_{\mathrm{B}}$, respectively, and $\beta_{\mathrm{A}}$ and $\beta_{\mathrm{B}}$ denote the total energies of $\mathscr{G}_{\mathrm{A}}$ and $\mathscr{G}_{\mathrm{B}}$, respectively, then the maximum entropy of $\mathscr{G}_{\mathrm{A}}$ and $\mathscr{G}_{\mathrm{B}}$ individually is achieved at $E_{\mathrm{A}}^{*}=\left(\beta_{\mathrm{A}} / q_{\mathrm{A}}\right) \mathbf{e}$ and $E_{\mathrm{B}}^{*}=\left(\beta_{\mathrm{B}} / q_{\mathrm{B}}\right) \mathbf{e}$, respectively, while the maximum entropy of the composite system $\mathscr{G}_{\mathrm{C}}$ is achieved at $E_{\mathrm{C}}^{*}=\left(\left(\beta_{\mathrm{A}}+\beta_{\mathrm{B}}\right) /\left(q_{\mathrm{A}}+q_{\mathrm{B}}\right)\right)$ e. (viii) It follows from Theorem 3.14 that for a stable equilibrium state $E=(\beta / q) \mathbf{e}$, where $\beta \geq 0$ is the total energy of the system $\mathscr{G}$ and $q$ is the number of subsystems of $\mathscr{G}$, the entropy is totally defined by $\beta$ and $q$; that is, $\mathscr{S}(E)=q \log _{e}(c+\beta / q)-q \log _{e} c$. Dual criteria to the eight criteria outlined above can also be established for an analytic expression representing system ectropy.

\section{Temperature equipartition}

The thermodynamic axioms introduced in Section 3 postulate that subsystem energies are synonymous to subsystem temperatures. In this section, we generalize the results of Section 3 to the case where the subsystem energies are proportional to the subsystem temperatures with the proportionality constants representing the subsystem specific heats or thermal capacities. In the case where the specific heats of all the subsystems are equal, the results of this section specialize to those of Section 3. To include temperature notions in our discrete-time large-scale dynamical system model, we replace Axioms 1, 2, and 3 of Section 3 by the following conditions. Let $\beta_{i}>0, i=1, \ldots, q$, denote the reciprocal of the specific heat of the $i$ th subsystem $\mathscr{G}_{i}$ so that the absolute temperature in $i$ th subsystem is given by $\hat{T}_{i}=\beta_{i} E_{i}$.

Axıом 1. For the connectivity matrix $\mathscr{b} \in \mathbb{R}^{q \times q}$ associated with the discrete-time large-scale dynamical system $\mathscr{G}$ defined by (3.12), $\operatorname{rank} \mathscr{C}=q-1$ and for $\mathscr{C}_{(i, j)}=1, i \neq j, \phi_{i j}(E)=0$ if and only if $\beta_{i} E_{i}=\beta_{j} E_{j}$.

Axiom 2. For $i, j=1, \ldots, q,\left(\beta_{i} E_{i}-\beta_{j} E_{j}\right) \phi_{i j}(E) \leq 0, E \in \overline{\mathbb{R}}_{+}^{q}$.

Axıом 3. For $i, j=1, \ldots, q,\left(\beta_{i} \Delta E_{i}-\beta_{j} \Delta E_{j}\right) /\left(\beta_{i} E_{i}-\beta_{j} E_{j}\right) \geq-1, \beta_{i} E_{i} \neq \beta_{j} E_{j}$.

Axiom 1 implies that if the temperatures in the connected subsystems $\mathscr{G}_{i}$ and $\mathscr{G}_{j}$ are equal, then heat exchange between these subsystems is not possible. This statement is consistent with the zeroth law of thermodynamics which postulates that temperature equality is a necessary and sufficient condition for thermal equilibrium. Axiom 2 implies that heat (energy) must flow in the direction of lower temperatures. This statement is consistent with the second law of thermodynamics which states that a transformation whose only final result is to transfer heat from a body at a given temperature to a body at a higher temperature is impossible. Axiom 3 implies that for any pair of connected subsystems $\mathscr{G}_{i}$ and $\mathscr{G}_{j}, i \neq j$, the temperature difference between consecutive time instants is monotonic; that is, $\left[\beta_{i} E_{i}(k+1)-\beta_{j} E_{j}(k+1)\right]\left[\beta_{i} E_{i}(k)-\beta_{j} E_{j}(k)\right] \geq 0$ for all $\beta_{i} E_{i} \neq \beta_{j} E_{j}, k \geq k_{0}$, $i, j=1, \ldots, q$. Next, in light of our modified conditions, we give a generalized definition for the entropy and ectropy of $\mathscr{G}$. The following proposition is needed for the statement of the main results of this section.

Proposition 4.1. Consider the discrete-time large-scale dynamical system $G$ with energy balance equation (3.2) and assume that Axioms 1, 2, and 3 hold. Then for all $E_{0} \in \overline{\mathbb{R}}_{+}^{q}$, 
$k_{\mathrm{f}} \geq k_{0}$, and $S(\cdot) \in \mathcal{U}$, such that $E\left(k_{\mathrm{f}}\right)=E\left(k_{0}\right)=E_{0}$,

$$
\begin{gathered}
\sum_{k=k_{0}}^{k_{\mathrm{f}}-1} \sum_{i=1}^{q} \frac{S_{i}(k)-\sigma_{i i}(E(k))}{c+\beta_{i} E_{i}(k+1)}=\sum_{k=k_{0}}^{k_{\mathrm{f}}-1} \sum_{i=1}^{q} \frac{Q_{i}(k)}{c+\beta_{i} E_{i}(k+1)} \leq 0, \\
\sum_{k=k_{0}}^{k_{\mathrm{f}}-1} \sum_{i=1}^{q} \beta_{i} E_{i}(k+1)\left[S_{i}(k)-\sigma_{i i}(E(k))\right]=\sum_{k=k_{0}}^{k_{\mathrm{f}}-1} \sum_{i=1}^{q} \beta_{i} E_{i}(k+1) Q_{i}(k) \geq 0,
\end{gathered}
$$

where $E(k), k \geq k_{0}$, is the solution to (3.2) with initial condition $E\left(k_{0}\right)=E_{0}$. Furthermore, equalities hold in (4.1) and (4.2) if and only if $\Delta E_{i}=0$ and $\beta_{i} E_{i}=\beta_{j} E_{j}, i, j=1, \ldots, q, i \neq j$.

Proof. The proof is identical to the proofs of Propositions 3.3 and 3.9.

Note that with the modified Axiom 1, the isolated discrete-time large-scale dynamical system $\mathscr{G}$ has equilibrium energy states given by $E_{\mathrm{e}}=\alpha \mathbf{p}$, for $\alpha \geq 0$, where $\mathbf{p} \triangleq\left[1 / \beta_{1}, \ldots\right.$, $\left.1 / \beta_{q}\right]^{\mathrm{T}}$. As in Section 3, we define an equilibrium process as a process where the trajectory of the system $\mathscr{G}$ stays at the equilibrium point of the isolated system $\mathscr{G}$ and a nonequilibrium process as a process that is not an equilibrium one. Thus, it follows from Axioms 1,2 , and 3 that inequalities (4.1) and (4.2) are satisfied as equalities for an equilibrium process and as strict inequalities for a nonequilibrium process.

Definition 4.2. For the discrete-time large-scale dynamical system $\mathscr{G}$ with energy balance equation (3.2), a function $\mathscr{S}: \overline{\mathbb{R}}_{+}^{q} \rightarrow \mathbb{R}$ satisfying

$$
\mathscr{S}\left(E\left(k_{2}\right)\right) \geq \mathscr{S}\left(E\left(k_{1}\right)\right)+\sum_{k=k_{1}}^{k_{2}-1} \sum_{i=1}^{q} \frac{S_{i}(k)-\sigma_{i i}(E(k))}{c+\beta_{i} E_{i}(k+1)},
$$

for any $k_{2} \geq k_{1} \geq k_{0}$ and $S(\cdot) \in \mathcal{U}$, is called the entropy of $\mathscr{G}$.

Definition 4.3. For the discrete-time large-scale dynamical system $\mathscr{G}$ with energy balance equation (3.2), a function $\mathscr{E}: \overline{\mathbb{R}}_{+}^{q} \rightarrow \mathbb{R}$ satisfying

$$
\mathscr{E}\left(E\left(k_{2}\right)\right) \leq \mathscr{E}\left(E\left(k_{1}\right)\right)+\sum_{k=k_{1}}^{k_{2}-1} \sum_{i=1}^{q} \beta_{i} E_{i}(k+1)\left[S_{i}(k)-\sigma_{i i}(E(k))\right]
$$

for any $k_{2} \geq k_{1} \geq k_{0}$ and $S(\cdot) \in \mathcal{U}$, is called the ectropy of $\mathscr{G}$.

For the next result, define the available entropy and available ectropy of the large-scale dynamical system $\mathscr{G}$ by

$$
\begin{gathered}
\mathscr{Y}_{\mathrm{a}}\left(E_{0}\right) \triangleq-\sup _{S(\cdot) \in \mathcal{U}_{\mathrm{c}}, K \geq k_{0}} \sum_{k=k_{0}}^{K-1} \sum_{i=1}^{q} \frac{S_{i}(k)-\sigma_{i i}(E(k))}{c+\beta_{i} E_{i}(k+1)}, \\
\mathscr{E}_{\mathrm{a}}\left(E_{0}\right) \triangleq-\inf _{S(\cdot) \in \mathcal{U}_{c}, K \geq k_{0}} \sum_{k=k_{0}}^{K-1} \sum_{i=1}^{q} \beta_{i} E_{i}(k+1)\left[S_{i}(k)-\sigma_{i i}(E(k))\right],
\end{gathered}
$$


where $E\left(k_{0}\right)=E_{0} \in \overline{\mathbb{R}}_{+}^{q}$ and $E(K)=0$, and define the required entropy supply and required ectropy supply of the large-scale dynamical system $\mathscr{G}$ by

$$
\begin{gathered}
\mathscr{S}_{\mathrm{r}}\left(E_{0}\right) \triangleq \sup _{S(\cdot) \in U_{\mathrm{r}}, K \geq-k_{0}+1} \sum_{k=-K}^{k_{0}-1} \sum_{i=1}^{q} \frac{S_{i}(k)-\sigma_{i i}(E(k))}{c+\beta_{i} E_{i}(k+1)}, \\
\mathscr{E}_{\mathrm{r}}\left(E_{0}\right) \triangleq \inf _{S(\cdot) \in U_{\mathrm{r}}, K \geq-k_{0}+1} \sum_{k=-K}^{k_{0}-1} \sum_{i=1}^{q} \beta_{i} E_{i}(k+1)\left[S_{i}(k)-\sigma_{i i}(E(k))\right],
\end{gathered}
$$

where $E(-K)=0$ and $E\left(k_{0}\right)=E_{0} \in \overline{\mathbb{R}}_{+}^{q}$.

Theorem 4.4. Consider the discrete-time large-scale dynamical system $\mathcal{G}$ with energy balance equation (3.2) and assume that Axioms 2 and 3 hold. Then there exist an entropy and

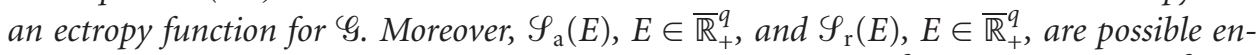
tropy functions for $\mathscr{G}_{\text {with }} \mathscr{S}_{\mathrm{a}}(0)=\mathscr{Y}_{\mathrm{r}}(0)=0$, and $\mathscr{E}_{\mathrm{a}}(E), E \in \overline{\mathbb{R}}_{+}^{q}$, and $\mathscr{E}_{\mathrm{r}}(E), E \in \overline{\mathbb{R}}_{+}^{q}$, are possible ectropy functions for $\mathscr{G}_{\text {with }} \mathscr{E}_{\mathrm{a}}(0)=\mathscr{E}_{\mathrm{r}}(0)=0$. Finally, all entropy functions $\mathscr{S}(E)$, $E \in \overline{\mathbb{R}}_{+}^{q}$, for $\varphi_{\text {satisfy }}$

$$
\mathscr{Y}_{\mathrm{r}}(E) \leq \mathscr{S}(E)-\mathscr{S}(0) \leq \mathscr{Y}_{\mathrm{a}}(E), \quad E \in \overline{\mathbb{R}}_{+}^{q},
$$

and all ectropy functions $\mathscr{E}(E), E \in \overline{\mathbb{R}}_{+}^{q}$, for $\mathscr{G}_{\text {satisfy }}$

$$
\mathscr{E}_{\mathrm{a}}(E) \leq \mathscr{E}(E)-\mathscr{E}(0) \leq \mathscr{E}_{\mathrm{r}}(E), \quad E \in \overline{\mathbb{R}}_{+}^{q}
$$

Proof. The proof is identical to the proofs of Theorems 3.5 and 3.11 .

For the statement of the next result, recall the definition of $\mathbf{p}=\left[1 / \beta_{1}, \ldots, 1 / \beta_{q}\right]^{\mathrm{T}}$ and define $P \triangleq \operatorname{diag}\left[\beta_{1}, \ldots, \beta_{q}\right]$.

Proposition 4.5. Consider the discrete-time large-scale dynamical system $\mathscr{G}$ with energy balance equation (3.2) and assume that Axioms 1, 2, and 3 hold. Then the function $\mathscr{S}$ : $\overline{\mathbb{R}}_{+}^{q} \rightarrow \mathbb{R}$ given by

$$
\mathscr{S}(E)=\mathbf{p}^{\mathrm{T}} \log _{e}(c \mathbf{e}+P E)-\mathbf{e}^{\mathrm{T}} \mathbf{p} \log _{e} c, \quad E \in \overline{\mathbb{R}}_{+}^{q},
$$

where $\log _{e}(c \mathbf{e}+P E)$ denotes the vector natural logarithm given by $\left[\log _{e}\left(c+\beta_{1} E_{1}\right), \ldots\right.$,

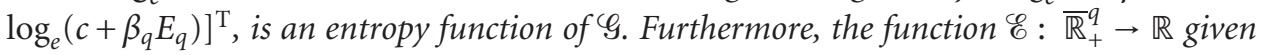
by

$$
\mathscr{E}(E)=\frac{1}{2} E^{\mathrm{T}} P E, \quad E \in \overline{\mathbb{R}}_{+}^{q},
$$

is an ectropy function of $\mathscr{G}$.

Proof. The proof is identical to the proofs of Propositions 3.7 and 3.12. 
Remark 4.6. As in Section 3, it can be shown that the entropy and ectropy functions for $G$ defined by (4.9) and (4.10) satisfy, respectively, (4.3) and (4.4) as equalities for an equilibrium process and as strict inequalities for a nonequilibrium process.

Once again, inequality (4.3) is analogous to Clausius' inequality for reversible and irreversible thermodynamics, while inequality (4.4) is an anti-Clausius' inequality. Moreover, for the ectropy function given by (4.10), inequality (4.4) shows that a thermodynamically consistent large-scale dynamical system model is dissipative with respect to the supply rate $E^{\mathrm{T}} P S$ and with storage function corresponding to the system ectropy $\mathscr{E}(E)$. In addition, if we let $Q_{i}(k)=S_{i}(k)-\sigma_{i i}(E(k)), i=1, \ldots, q$, denote the net amount of heat received or dissipated by the ith subsystem of $\mathscr{G}$ at a given time instant at the (shifted) absolute ith subsystem temperature $T_{i} \triangleq c+\beta_{i} E_{i}$, then it follows from (4.3) that the system entropy varies by an amount

$$
\Delta \mathscr{S}(E(k)) \geq \sum_{i=1}^{q} \frac{Q_{i}(k)}{c+\beta_{i} E_{i}(k+1)}, \quad k \geq k_{0} .
$$

Finally, note that the nonconservation of entropy and ectropy equations (3.47) and (3.48), respectively, for isolated discrete-time large-scale dynamical systems also hold for the more general definitions of entropy and ectropy given in Definitions 4.2 and 4.3. The following theorem is a generalization of Theorem 3.14.

Theorem 4.7. Consider the discrete-time large-scale dynamical system $\mathcal{G}$ with energy balance equation (3.2) with $S(k) \equiv 0$ and $d(E) \equiv 0$, and assume that Axioms 1, 2, and 3 hold. Then for every $\alpha \geq 0, \alpha \mathbf{p}$ is a semistable equilibrium state of (3.2). Furthermore, $E(k)$ $\rightarrow\left(1 / \mathbf{e}^{\mathrm{T}} \mathbf{p}\right) \mathbf{p} \mathbf{e}^{\mathrm{T}} E\left(k_{0}\right)$ as $k \rightarrow \infty$ and $\left(1 / \mathbf{e}^{\mathrm{T}} \mathbf{p}\right) \mathbf{p e}^{\mathrm{T}} E\left(k_{0}\right)$ is a semistable equilibrium state. Finally, if for some $m \in\{1, \ldots, q\}, \sigma_{m m}(E) \geq 0$, and $\sigma_{m m}(E)=0$ if and only if $E_{m}=0$, then the zero solution $E(k) \equiv 0$ to (3.2) is a globally asymptotically stable equilibrium state of (3.2).

Proof. It follows from Axiom 1 that $\alpha \mathbf{p} \in \overline{\mathbb{R}}_{+}^{q}, \alpha \geq 0$, is an equilibrium state for (3.2). To show Lyapunov stability of the equilibrium state $\alpha \mathbf{p}$, consider the system shifted ectropy $\mathscr{E}_{s}(E)=(1 / 2)(E-\alpha \mathbf{p})^{\mathrm{T}} P(E-\alpha \mathbf{p})$ as a Lyapunov function candidate. Now, the proof follows as in the proof of Theorem 3.14 by invoking Axioms 1, 2, and 3, and noting that $\phi_{i j}(E)=-\phi_{j i}(E), E \in \overline{\mathbb{R}}_{+}^{q}, i \neq j, i, j=1, \ldots, q, P \mathbf{p}=\mathbf{e}$, and $\mathbf{e}^{\mathrm{T}} w(E)=\mathbf{e}^{\mathrm{T}} E, E \in \overline{\mathbb{R}}_{+}^{q}$. Alternatively, in the case where for some $m \in\{1, \ldots, q\}, \sigma_{m m}(E) \geq 0$, and $\sigma_{m m}(E)=0$ if and only if $E_{m}=0$, global asymptotic stability of the zero solution $E(k) \equiv 0$ to (3.2) follows from standard Lyapunov arguments using the system ectropy $\mathscr{E}(E)=(1 / 2) E^{\mathrm{T}} P E$ as a candidate Lyapunov function.

It follows from Theorem 4.7 that the steady-state value of the energy in each subsystem $\mathscr{G}_{i}$ of the isolated discrete-time large-scale dynamical system $\mathscr{G}_{\text {is }}$ given by $E_{\infty}=$ $\left(1 / \mathbf{e}^{\mathrm{T}} \mathbf{p}\right) \mathbf{p} \mathbf{e}^{\mathrm{T}} E\left(k_{0}\right)$, which implies that $E_{i \infty}=\left(1 / \beta_{i} \mathbf{e}^{\mathrm{T}} \mathbf{p}\right) \mathbf{e}^{\mathrm{T}} E\left(k_{0}\right)$ or, equivalently, $\hat{T}_{i \infty}=$ $\beta_{i} E_{i \infty}=\left(1 / \mathbf{e}^{\mathrm{T}} \mathbf{p}\right) \mathbf{e}^{\mathrm{T}} E\left(k_{0}\right)$. Hence, the steady-state temperature of the isolated discretetime large-scale dynamical system $\mathscr{G}$ given by $\widehat{T}_{\infty}=\left(1 / \mathbf{e}^{\mathrm{T}} \mathbf{p}\right) \mathbf{e}^{\mathrm{T}} E\left(k_{0}\right) \mathbf{e}$ is uniformly distributed over all the subsystems of $\mathscr{G}$. This phenomenon is known as temperature 
equipartition in which all the system energy is eventually transformed into heat at a uniform temperature, and hence all system motion would cease.

Proposition 4.8. Consider the discrete-time large-scale dynamical system $\mathscr{G}$ with energy balance equation (3.2), let $\mathscr{E}: \overline{\mathbb{R}}_{+}^{q} \rightarrow \overline{\mathbb{R}}_{+}$and $\mathscr{S}: \overline{\mathbb{R}}_{+}^{q} \rightarrow \mathbb{R}$ denote the ectropy and entropy of $G$ and be given by (4.10) and (4.9), respectively, and define $\mathscr{D}_{\mathrm{c}} \triangleq\left\{E \in \overline{\mathbb{R}}_{+}^{q}: \mathrm{e}^{\mathrm{T}} E=\beta\right\}$, where $\beta \geq 0$. Then,

$$
\underset{E \in \mathscr{D}_{\mathrm{c}}}{\arg \min }(\mathscr{E}(E))=\underset{E \in \mathscr{D}_{\mathrm{c}}}{\arg \max }(\mathscr{Y}(E))=E^{*}=\frac{\beta}{\mathbf{e}^{\mathrm{T}} \mathbf{p}} \mathbf{p} .
$$

Furthermore, $\mathscr{E}_{\min } \triangleq \mathscr{E}\left(E^{*}\right)=(1 / 2)\left(\beta^{2} / \mathbf{e}^{\mathrm{T}} \mathbf{p}\right)$ and $\mathscr{I}_{\max } \triangleq \mathscr{S}\left(E^{*}\right)=\mathbf{e}^{\mathrm{T}} \mathbf{p} \log _{e}\left(c+\beta / \mathbf{e}^{\mathrm{T}} \mathbf{p}\right)$ $-\mathbf{e}^{\mathrm{T}} \mathbf{p} \log _{e} c$.

Proof. The proof is identical to the proof of Proposition 3.17, and hence is omitted.

Proposition 4.8 shows that when all the energy of a discrete-time large-scale dynamical system is transformed into heat at a uniform temperature, entropy is a maximum and ectropy is a minimum.

Next, we provide an interpretation of the (steady-state) expressions for entropy and ectropy presented in this section that is consistent with kinetic theory. Specifically, we assume that each subsystem $\mathscr{G}_{i}$ of the discrete-time large-scale dynamical system $\mathscr{G}_{\text {is }}$ a simple system consisting of an ideal gas with rigid walls. Furthermore, we assume that all subsystems $\mathscr{G}_{i}$ are divided by diathermal walls (i.e., walls that permit energy flow) and the overall dynamical system is a closed system; that is, the system is separated from the environment by a rigid adiabatic wall. In this case, $\beta_{i}=k / n_{i}, i=1, \ldots, q$, where $n_{i}$, $i=1, \ldots, q$, is the number of molecules in the $i$ th subsystem and $k>0$ is the Boltzmann constant (i.e., gas constant per molecule). Without loss of generality and for simplicity of exposition, let $k=1$. In addition, we assume that the molecules in the ideal gas are hard elastic spheres; that is, there are no forces between the molecules except during collisions and the molecules are not deformed by collisions. Thus, there is no internal potential energy and the system internal energy of the ideal gas is entirely kinetic. Hence, in this case, the temperature of each subsystem $\mathscr{G}_{i}$ is the average translational kinetic energy per molecule which is consistent with the kinetic theory of ideal gases.

Definition 4.9. For a given isolated discrete-time large-scale dynamical system $\mathscr{G}$ in thermal equilibrium, define the equilibrium entropy of $\varphi_{\text {by }} \mathscr{S}_{\mathrm{e}}=n \log _{e}\left(c+\mathrm{e}^{\mathrm{T}} E_{\infty} / n\right)-n \log _{e} c$

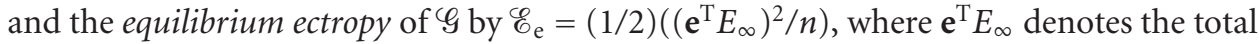
steady-state energy of the discrete-time large-scale dynamical system $\mathscr{G}_{\text {and }} n$ denotes the number of molecules in $\mathscr{G}$.

Note that the equilibrium entropy and ectropy in Definition 4.9 is entirely consistent with the equilibrium (maximum) entropy and equilibrium (minimum) ectropy given by Proposition 4.8. Next, assume that each subsystem $\mathscr{G}_{i}$ is initially in thermal equilibrium. Furthermore, for each subsystem, let $E_{i}$ and $n_{i}, i=1, \ldots, q$, denote the total internal energy and the number of molecules, respectively, in the $i$ th subsystem. Hence, the entropy 
and ectropy of the $i$ th subsystem are given by $\mathscr{S}_{i}=n_{i} \log _{e}\left(c+E_{i} / n_{i}\right)-n_{i} \log _{e} c$ and $\mathscr{E}_{i}=$ $(1 / 2)\left(E_{i}^{2} / n_{i}\right)$, respectively. Next, note that the entropy and the ectropy of the overall system (after reaching a thermal equilibrium) are given by $\mathscr{S}_{\mathrm{e}}=n \log _{e}\left(c+\mathrm{e}^{\mathrm{T}} E_{\infty} / n\right)-n \log _{e} c$ and $\mathscr{E}_{\mathrm{e}}=(1 / 2)\left(\left(\mathbf{e}^{\mathrm{T}} E_{\infty}\right)^{2} / n\right)$. Now, it follows from the convexity of $-\log _{e}(\cdot)$ and conservation of energy that the entropy of $\mathscr{G}$ at thermal equilibrium is given by

$$
\begin{aligned}
\mathscr{S}_{\mathrm{e}} & =n \log _{e}\left(c+\frac{\mathrm{e}^{\mathrm{T}} E_{\infty}}{n}\right)-n \log _{e} c \\
& =n \log _{e}\left[\sum_{i=1}^{q} \frac{n_{i}}{n}\left(c+\frac{E_{i}}{n_{i}}\right)\right]-\sum_{i=1}^{q} n_{i} \log _{e} c \\
& \geq n \sum_{i=1}^{q} \frac{n_{i}}{n} \log _{e}\left(c+\frac{E_{i}}{n_{i}}\right)-\sum_{i=1}^{q} n_{i} \log _{e} c \\
& =\sum_{i=1}^{q} \mathscr{S}_{i} .
\end{aligned}
$$

Furthermore, the ectropy of $\mathscr{G}$ at thermal equilibrium is given by

$$
\begin{aligned}
\mathscr{E}_{\mathrm{e}} & =\frac{1}{2} \frac{\left(\mathbf{e}^{\mathrm{T}} E_{\infty}\right)^{2}}{n} \\
& =\sum_{i=1}^{q} \frac{1}{2} \frac{E_{i}^{2}}{n_{i}}-\frac{1}{2 n} \sum_{i=1}^{q-1} \sum_{j=i+1}^{q} \frac{\left(n_{j} E_{i}-n_{i} E_{j}\right)^{2}}{n_{i} n_{j}} \\
& \leq \sum_{i=1}^{q} \frac{1}{2} \frac{E_{i}^{2}}{n_{i}} \\
& =\sum_{i=1}^{q} \mathscr{E}_{i} .
\end{aligned}
$$

It follows from (4.13) (resp., (4.14)) that the equilibrium entropy (resp., ectropy) of the system (gas) $\mathscr{G}$ is always greater (resp., less) than or equal to the sum of entropies (resp., ectropies) of the individual subsystems $\mathscr{G}_{i}$. Hence, the entropy (resp., ectropy) of the gas increases (resp., decreases) as a more evenly distributed (disordered) state is reached. Finally, note that it follows from (4.13) and (4.14) that $\mathscr{Y}_{\mathrm{e}}=\sum_{i=1}^{q} \mathscr{Y}_{i}$ and $\mathscr{E}_{\mathrm{e}}=\sum_{i=1}^{q} \mathscr{E}_{i}$ if and only if $E_{i} / n_{i}=E_{j} / n_{j}, i \neq j, i, j=1, \ldots, q$; that is, the initial temperatures of all subsystems are equal.

\section{Thermodynamic models with linear energy exchange}

In this section, we specialize the results of Section 3 to the case of large-scale dynamical systems with linear energy exchange between subsystems; that is, $w(E)=W E$ and $d(E)=$ $D E$, where $W \in \mathbb{R}^{q \times q}$ and $D \in \mathbb{R}^{q \times q}$. In this case, the vector form of the energy balance 
equation (3.2), with $k_{0}=0$, is given by

$$
E(k+1)=W E(k)-D E(k)+S(k), \quad E(0)=E_{0}, \quad k \geq 0 .
$$

Next, let the net energy exchange from the $j$ th subsystem $\mathscr{G}_{j}$ to the $i$ th subsystem $\mathscr{G}_{i}$ be parameterized as $\phi_{i j}(E)=\Phi_{i j}^{\mathrm{T}} E$, where $\Phi_{i j} \in \mathbb{R}^{q}$ and $E \in \overline{\mathbb{R}}_{+}^{q}$. In this case, since $w_{i}(E)=$ $E_{i}+\sum_{i=1, j \neq i}^{q} \phi_{i j}(E)$, it follows that

$$
W=I_{q}+\left[\sum_{j=2}^{q} \Phi_{1 j}, \ldots, \sum_{j=1, j \neq i}^{q} \Phi_{i j}, \ldots, \sum_{j=1}^{q-1} \Phi_{q j}\right]^{\mathrm{T}}
$$

Since $\phi_{i j}(E)=-\phi_{j i}(E), i, j=1, \ldots, q, i \neq j, E \in \overline{\mathbb{R}}_{+}^{q}$, it follows that $\Phi_{i j}=-\Phi_{j i}, i \neq j, i, j=$ $1, \ldots, q$. The following proposition considers the special case where $W$ is symmetric.

Proposition 5.1. Consider the large-scale dynamical system $G$ with energy balance equation given by (5.1) and with $D=0$. Then Axioms 1 and 2 hold if and only if $W=W^{\mathrm{T}}$, $\left(W-I_{q}\right) \mathbf{e}=0, \operatorname{rank}\left(W-I_{q}\right)=q-1$, and $W$ is nonnegative. In addition, if $S=0$ and Axiom 3 holds, then $\operatorname{rank}\left(W+I_{q}\right)=q$ and $\operatorname{rank}\left(W^{2}-I_{q}\right)=q-1$.

Proof. Assume that Axioms 1 and 2 hold. Since, by Axiom 2, $\left(E_{i}-E_{j}\right) \phi_{i j}(E) \leq 0, E \in \overline{\mathbb{R}}_{+}^{q}$, it follows that $E^{\mathrm{T}} \Phi_{i j} \mathbf{e}_{i j}^{\mathrm{T}} E \leq 0, i, j=1, \ldots, q, i \neq j$, where $E \in \overline{\mathbb{R}}_{+}^{q}$ and $\mathbf{e}_{i j} \in \mathbb{R}^{q}$ is a vector whose $i$ th entry is $1, j$ th entry is -1 , and remaining entries are zero. Next, it can be shown that $E^{\mathrm{T}} \Phi_{i j} \mathbf{e}_{i j}^{\mathrm{T}} E \leq 0, E \in \overline{\mathbb{R}}_{+}^{q}, i \neq j, i, j=1, \ldots, q$, if and only if $\Phi_{i j} \in \mathbb{R}^{q}$ is such that its $i$ th entry is $-\sigma_{i j}$, its $j$ th entry is $\sigma_{i j}$, and its remaining entries are zero, where $\sigma_{i j} \geq 0$. Furthermore, since $\Phi_{i j}=-\Phi_{j i}, i \neq j, i, j=1, \ldots, q$, it follows that $\sigma_{i j}=\sigma_{j i}, i \neq j$, $i, j=1, \ldots, q$. Hence, $W$ is given by

$$
W_{(i, j)}= \begin{cases}1-\sum_{k=1, k \neq j}^{q} \sigma_{k j}, & i=j, \\ \sigma_{i j}, & i \neq j,\end{cases}
$$

which implies that $W$ is symmetric ( since $\left.\sigma_{i j}=\sigma_{j i}\right)$ and $\left(W-I_{q}\right) \mathbf{e}=0$. Note that since at any given instant of time, energy can only be transported or stored but not created and the maximum amount of energy that can be transported cannot exceed the energy in a compartment, it follows that $1 \geq \sum_{k=1, k \neq j}^{q} \sigma_{k j}$. Thus, $W$ is a nonnegative matrix. Now, since by Axiom $1, \phi_{i j}(E)=0$ if and only if $E_{i}=E_{j}$ for all $i, j=1, \ldots, q, i \neq j$, such that $\mathscr{C}_{(i, j)}=1$, it follows that $\sigma_{i j}>0$ for all $i, j=1, \ldots, q, i \neq j$, such that $\mathscr{C}_{(i, j)}=1$. Hence, $\operatorname{rank}\left(W-I_{q}\right)=\operatorname{rank} \mathscr{C}=q-1$. The converse is immediate and hence is omitted.

Next, assume that Axiom 3 holds. Since, by Axiom 3, $\left(E_{i}(k+1)-E_{j}(k+1)\right)\left(E_{i}(k)-\right.$ $\left.E_{j}(k)\right) \geq 0, i, j=1, \ldots, q, i \neq j, k \geq k_{0}$, it follows that $E^{\mathrm{T}}(k+1) \mathbf{e}_{i j} \mathbf{e}_{i j}^{\mathrm{T}} E(k) \geq 0$ or, equivalently, $E^{\mathrm{T}}(k) W^{\mathrm{T}} \mathbf{e}_{i j} \mathbf{e}_{i j}^{\mathrm{T}} E(k) \geq 0, i, j=1, \ldots, q, i \neq j, k \geq k_{0}$, where $E \in \overline{\mathbb{R}}_{+}^{q}$. Next, we show that $I_{q}+W$ is strictly diagonally dominant. Suppose, ad absurdum, that $1+W_{(i, i)} \leq$ $\sum_{l=1, l \neq i}^{q} W_{(i, l)}$ for some $i, 1 \leq i \leq q$. Let $E\left(k_{0}\right)=\mathbf{e}_{i}, i=1, \ldots, q$, where $\mathbf{e}_{i} \in \overline{\mathbb{R}}_{+}^{q}$ is a vector 
whose $i$ th entry is 1 and remaining entries are zero. Then,

$$
\begin{aligned}
E^{\mathrm{T}}\left(k_{0}\right) W^{\mathrm{T}} \mathbf{e}_{i j} \mathbf{e}_{i j}^{\mathrm{T}} E\left(k_{0}\right) & =\mathbf{e}_{i}^{\mathrm{T}} W^{\mathrm{T}} \mathbf{e}_{i j} \mathbf{e}_{i j}^{\mathrm{T}} \mathbf{e}_{i} \\
& =W_{(i, i)}-W_{(i, j)} \\
& =1-\sum_{k=1, k \neq j}^{q} \sigma_{k j}-\sigma_{i j} \\
& \geq 0, \quad i, j=1, \ldots, q, i \neq j .
\end{aligned}
$$

Now, it follows from (5.4) that

$$
1+W_{(i, j)} \leq 1+W_{(i, i)} \leq \sum_{l=1, l \neq i}^{q} W_{(i, l)}, \quad j=1, \ldots, q, j \neq i, 1 \leq i \leq q,
$$

or, equivalently,

$$
1 \leq \sum_{l=1, l \neq i, l \neq j}^{q} W_{(i, l)}, \quad j=1, \ldots, q, j \neq i, 1 \leq i \leq q .
$$

However, since $W$ is compartmental and symmetric, it follows that

$$
\sum_{l=1, l \neq i}^{q} W_{(i, l)}=\sum_{l=1, l \neq i}^{q} W_{(l, i)}=\sum_{l=1, l \neq i}^{q} \sigma_{l, i} \leq 1, \quad i=1, \ldots, q .
$$

Now, since $W_{(i, j)}=\sigma_{i j}>0$ for all $i, j=1, \ldots, q, i \neq j$, it follows that

$$
\sum_{l=1, l \neq i, l \neq j}^{q} W_{(i, l)}<\sum_{l=1, l \neq i}^{q} W_{(i, l)} \leq 1, \quad i=1, \ldots, q,
$$

which contradicts (5.6).

Next, since $I_{q}+W$ is strictly diagonally dominant it follows from [15, Theorem 6.1.10] that $\operatorname{rank}\left(I_{q}+W\right)=q$. Furthermore, since $\operatorname{rank}\left(W^{2}-I_{q}\right)=\operatorname{rank}\left(W+I_{q}\right)\left(W-I_{q}\right)$, it follows from Sylvester's inequality that

$$
\begin{aligned}
\operatorname{rank}\left(W+I_{q}\right)+\operatorname{rank}\left(W-I_{q}\right)-q & \leq \operatorname{rank}\left(W^{2}-I_{q}\right) \\
& \leq \min \left\{\operatorname{rank}\left(W+I_{q}\right), \operatorname{rank}\left(W-I_{q}\right)\right\} .
\end{aligned}
$$

Now, $\operatorname{rank}\left(W^{2}-I_{q}\right)=q-1$ follows from (5.9) by noting that $\operatorname{rank}\left(W-I_{q}\right)=q-1$ and $\operatorname{rank}\left(W+I_{q}\right)=q$. 
Next, we specialize the energy balance equation (5.1) to the case where $D=\operatorname{diag}\left[\sigma_{11}\right.$, $\left.\sigma_{22}, \ldots, \sigma_{q q}\right]$. In this case, the vector form of the energy balance equation (3.2), with $k_{0}=0$, is given by

$$
E(k+1)=A E(k)+S(k), \quad E(0)=E_{0}, k \in \overline{\mathbb{Z}}_{+},
$$

where $A \triangleq W-D$ is such that

$$
A_{(i, j)}= \begin{cases}1-\sum_{k=1}^{q} \sigma_{k j}, & i=j, \\ \sigma_{i j}, & i \neq j .\end{cases}
$$

Note that (5.11) implies that $\sum_{i=1}^{q} A_{(i, j)}=1-\sigma_{i i} \leq 1, j=1, \ldots, q$, and hence $A$ is a Lyapunov stable compartmental matrix. If $\sigma_{i i}>0, i=1, \ldots, q$, then $A$ is an asymptotically stable compartmental matrix.

An important special case of (5.10) is the case where $A$ is symmetric or, equivalently, $\sigma_{i j}=\sigma_{j i}, i \neq j, i, j=1, \ldots, q$. In this case, it follows from (5.10) that for each subsystem, the energy balance equation satisfies

$$
\Delta E_{i}(k)+\sigma_{i i} E_{i}(k)+\sum_{j=1, j \neq i}^{q} \sigma_{i j}\left[E_{i}(k)-E_{j}(k)\right]=S_{i}(k), \quad k \in \overline{\mathbb{Z}}_{+} .
$$

Note that $\phi_{i}(E) \triangleq \sum_{j=1, j \neq i}^{q} \sigma_{i j}\left(E_{i}-E_{j}\right), i=1, \ldots, q$, represents the energy exchange from the $i$ th subsystem to all other subsystems and is given by the sum of the individual energy exchanges from the $i$ th subsystem to the $j$ th subsystem. Furthermore, these energy exchanges are proportional to the energy differences of the subsystems; that is, $E_{i}-E_{j}$. Hence, (5.12) is an energy balance equation that governs the energy exchange among coupled subsystems and is completely analogous to the equations of thermal transfer with subsystem energies playing the role of temperatures. Furthermore, note that since $\sigma_{i j} \geq 0$, $i, j=1, \ldots, q$, energy is exchanged from more energetic subsystems to less energetic subsystems, which is consistent with the second law of thermodynamics which requires that heat (energy) must flow in the direction of lower temperatures.

The next lemma and proposition are needed for developing expressions for steadystate energy distributions of the discrete-time large-scale dynamical system $\mathscr{G}$ with linear energy balance equation (5.10).

Lemma 5.2. Let $A \in \mathbb{R}^{q \times q}$ be compartmental and let $S \in \mathbb{R}^{q}$. Then the following properties hold:

(i) $I_{q}-A$ is an $M$-matrix,

(ii) $|\lambda| \leq 1, \lambda \in \operatorname{spec}(A)$,

(iii) if $A$ is semistable and $\lambda \in \operatorname{spec}(A)$, then either $|\lambda|<1$ or $\lambda=1$ and $\lambda=1$ is semisimple,

(iv) $\operatorname{ind}\left(I_{q}-A\right) \leq 1$ and $\operatorname{ind}(A) \leq 1$,

(v) if $A$ is semistable, then $\lim _{k \rightarrow \infty} A^{k}=I_{q}-\left(A-I_{q}\right)\left(A-I_{q}\right)^{\#} \geq \geq 0$,

(vi) $\mathscr{R}\left(A-I_{q}\right)=\mathcal{N}\left(I_{q}-\left(A-I_{q}\right)\left(A-I_{q}\right)^{\#}\right)$ and $\mathcal{N}\left(A-I_{q}\right)=\mathscr{R}\left(I_{q}-\left(A-I_{q}\right)\left(A-I_{q}\right)^{\#}\right)$, 
(vii) $\sum_{i=0}^{k} A^{i}=\left(A-I_{q}\right)^{\#}\left(A^{k+1}-I_{q}\right)+(k+1)\left[I_{q}-\left(A-I_{q}\right)\left(A-I_{q}\right)^{\#}\right], k \in \overline{\mathbb{Z}}_{+}$,

(viii) if $A$ is semistable, then $\sum_{i=0}^{\infty} A^{i} S$ exists if and only if $S \in \mathscr{R}\left(A-I_{q}\right)$, where $S \in \mathbb{R}^{q}$,

(ix) if $A$ is semistable and $S \in \mathscr{R}\left(A-I_{q}\right)$, then $\sum_{i=0}^{\infty} A^{i} S=-\left(A-I_{q}\right)^{\#} S$,

(x) if $A$ is semistable, $S \in \mathscr{R}\left(A-I_{q}\right)$, and $S \geq \geq 0$, then $-\left(A-I_{q}\right)^{\#} S \geq \geq 0$,

(xi) $A-I_{q}$ is nonsingular if and only if $I_{q}-A$ is a nonsingular $M$-matrix,

(xii) if $A$ is semistable and $A-I_{q}$ is nonsingular, then $A$ is asymptotically stable and $\left(I_{q}-\right.$ $A)^{-1} \geq \geq 0$.

Proof. (i) Note that $A^{\mathrm{T}} \mathbf{e}=\left[-\left(1-\sum_{i=1}^{q} A_{(i, 1)}\right),-\left(1-\sum_{i=1}^{q} A_{(i, 2)}\right), \ldots,-\left(1-\sum_{i=1}^{q} A_{(i, q)}\right)\right]^{\mathrm{T}}+$ e. Then $\left(I_{q}-A\right)^{\mathrm{T}} \mathbf{e} \geq \geq 0$ and $I_{q}-A$ is a $Z$-matrix. It follows from [3, Theorem 1] that $\left(I_{q}-A\right)^{\mathrm{T}}$, and hence $I_{q}-A$ is an $M$-matrix.

(ii) The result follows from (i) and [12, Lemma 1].

(iii) The result follows from [12, Theorem 2].

(iv) Since $\left(I_{q}-A\right)^{\mathrm{T}} \mathbf{e} \geq \geq 0$, it follows that $I_{q}-A$ is an $M$-matrix and has "property c" (see [2]). Hence, it follows from [2, Lemma 4.11] that $I_{q}-A$ has "property c" if and only if ind $\left(I_{q}-A\right) \leq 1$. Next, since ind $\left(I_{q}-A\right) \leq 1$, it follows from the real Jordan decomposition that there exist invertible matrices $J \in \mathbb{R}^{r \times r}$, where $r=\operatorname{rank}\left(I_{q}-A\right)$, and $U \in \mathbb{R}^{q \times q}$ such that $J$ is diagonal and

$$
I_{q}-A=U\left[\begin{array}{ll}
J & 0 \\
0 & 0
\end{array}\right] U^{-1}
$$

which implies that

$$
A=U\left[\begin{array}{cc}
I_{r}-J & 0 \\
0 & I_{q-r}
\end{array}\right] U^{-1} .
$$

Hence, ind $(A) \leq 1$,

(v) The result follows from [12, Theorem 2].

(vi) Let $x \in \mathscr{R}\left(A-I_{q}\right)$; that is, there exists $y \in \mathbb{R}^{q}$ such that $x=\left(A-I_{q}\right) y$. Now, $\left(I_{q}-\right.$ $\left.\left(A-I_{q}\right)\left(A-I_{q}\right)^{\#}\right) x=x-\left(A-I_{q}\right)\left(A-I_{q}\right)^{\#}\left(A-I_{q}\right) y=x-\left(A-I_{q}\right) y=0$, which implies that $\mathscr{R}\left(A-I_{q}\right) \subseteq \mathcal{N}\left(I_{q}-\left(A-I_{q}\right)\left(A-I_{q}\right)^{\#}\right)$. Conversely, let $x \in \mathcal{N}\left(I_{q}-\left(A-I_{q}\right)\left(A-I_{q}\right)^{\#}\right)$. Hence, $\left(I_{q}-\left(A-I_{q}\right)\left(A-I_{q}\right)^{\#}\right) x=0$, or, equivalently, $x=\left(A-I_{q}\right)\left(A-I_{q}\right)^{\#} x$, which implies that $x \in \mathscr{R}\left(A-I_{q}\right)$ which proves that $\mathscr{R}\left(A-I_{q}\right)=\mathcal{N}\left(I_{q}-\left(A-I_{q}\right)\left(A-I_{q}\right)^{\#}\right)$. The equality $\mathcal{N}\left(A-I_{q}\right)=\mathscr{R}\left(I_{q}-\left(A-I_{q}\right)\left(A-I_{q}\right)^{\#}\right)$ can be proved in an analogous manner.

(vii) Note since $A=U\left[\begin{array}{cc}I_{r}-J & 0 \\ 0 & I_{q-r}\end{array}\right] U^{-1}$, and $J$ is invertible, it follows that

$$
\begin{aligned}
\sum_{i=0}^{k} A^{i} & =\sum_{i=0}^{k} U\left[\begin{array}{cc}
\left(I_{r}-J\right)^{i} & 0 \\
0 & I_{q-r}
\end{array}\right] U^{-1} \\
& =U\left[\begin{array}{cc}
\sum_{i=0}^{k}\left(I_{r}-J\right)^{i} & 0 \\
0 & (k+1) I_{q-r}
\end{array}\right] U^{-1}
\end{aligned}
$$


312 Thermodynamic modeling for discrete-time systems

$$
\begin{aligned}
= & U\left[\begin{array}{cc}
-J^{-1}\left[\left(I_{r}-J\right)^{k+1}-I_{r}\right] & 0 \\
0 & (k+1) I_{q-r}
\end{array}\right] U^{-1} \\
= & U\left[\begin{array}{cc}
-J^{-1} & 0 \\
0 & 0
\end{array}\right] U^{-1} U\left[\begin{array}{cc}
\left(I_{r}-J\right)^{k+1}-I_{r} & 0 \\
0 & 0
\end{array}\right] U^{-1}+U\left[\begin{array}{cc}
0 & 0 \\
0 & (k+1) I_{q-r}
\end{array}\right] U^{-1} \\
= & \left(A-I_{q}\right)^{\#}\left(A^{k+1}-I_{q}\right) \\
& +(k+1)\left(I_{q}-U\left[\begin{array}{cc}
J-I_{r} & 0 \\
0 & 0
\end{array}\right] U^{-1} U\left[\begin{array}{cc}
\left(J-I_{r}\right)^{-1} & 0 \\
0 & 0
\end{array}\right] U^{-1}\right) \\
= & \left(A-I_{q}\right)^{\#}\left(A^{k+1}-I_{q}\right)+(k+1)\left[I_{q}-\left(A-I_{q}\right)\left(A-I_{q}\right)^{\#}\right], \quad k \in \overline{\mathbb{Z}}_{+} .
\end{aligned}
$$

(viii) The result is a direct consequence of (v)-(vii).

(ix) The result follows from (v) and (vii).

(x) The result follows from (ix).

(xi) The result follows from (i).

(xii) Asymptotic stability of $A$ is a direct consequence of (iii). $\left(I_{q}-A\right)^{-1} \geq \geq 0$ follows from [12, Lemma 1].

Proposition 5.3 [12]. Consider the discrete-time large-scale dynamical system $\varphi$ with energy balance equation given by (5.10). Suppose that $E_{0} \geq \geq 0$, and $S(k) \geq \geq 0, k \in \overline{\mathbb{Z}}_{+}$. Then the solution $E(k), k \in \overline{\mathbb{Z}}_{+}$, to (5.10) is nonnegative for all $k \in \overline{\mathbb{Z}}_{+}$if and only if $A$ is nonnegative.

Next, we develop expressions for the steady-state energy distribution for a discretetime large-scale linear dynamical system $\mathscr{G}$ for the cases where $A$ is semistable, and the supplied system energy $S(k)$ is a periodic function with period $\tau \in \overline{\mathbb{Z}}_{+}, \tau>0$; that is, $S(k+$ $\tau)=S(k), k \in \overline{\mathbb{Z}}_{+}$, and $S(k)$ is constant; that is, $S(k) \equiv S$. Define $e(k) \triangleq E(k)-E(k+\tau)$, $k \in \overline{\mathbb{Z}}_{+}$, and note that

$$
e(k+1)=A e(k), \quad e(0)=E(0)-E(\tau), \quad k \in \overline{\mathbb{Z}}_{+} .
$$

Hence, since

$$
e(k)=A^{k}[E(0)-E(\tau)], \quad k \in \overline{\mathbb{Z}}_{+},
$$

and $A$ is semistable, it follows from (v) of Lemma 5.2 that

$$
\begin{aligned}
\lim _{k \rightarrow \infty} e(k) & =\lim _{k \rightarrow \infty}[E(k)-E(k+\tau)] \\
& =\left[I_{q}-\left(A-I_{q}\right)\left(A-I_{q}\right)^{\#}\right][E(0)-E(\tau)],
\end{aligned}
$$

which represents a constant offset to the steady-state error energy distribution in the discrete-time large-scale nonlinear dynamical system $\mathscr{G}$. For the case where $S(k) \equiv S$, $\tau \rightarrow \infty$, and hence the following result is immediate.

Proposition 5.4. Consider the discrete-time large-scale dynamical system $\mathscr{G}$ with energy balance equation given by (5.10). Suppose that $A$ is semistable, $E_{0} \geq \geq 0$, and $S(k) \equiv S \geq \geq 0$. 
Then $E_{\infty} \triangleq \lim _{k \rightarrow \infty} E(k)$ exists if and only if $S \in \mathscr{R}\left(A-I_{q}\right)$. In this case,

$$
E_{\infty}=\left[I_{q}-\left(A-I_{q}\right)\left(A-I_{q}\right)^{\#}\right] E_{0}-\left(A-I_{q}\right)^{\#} S
$$

and $E_{\infty} \geq \geq 0$. If, in addition, $A-I_{q}$ is nonsingular, then $E_{\infty}$ exists for all $S \geq \geq 0$ and is given by

$$
E_{\infty}=\left(I_{q}-A\right)^{-1} S
$$

Proof. Note that it follows from Lagrange's formula that the solution $E(k), k \in \overline{\mathbb{Z}}_{+}$, to (5.10) is given by

$$
E(k)=A^{k} E_{0}+\sum_{i=0}^{k-1} A^{(k-1-i)} S(i), \quad k \in \overline{\mathbb{Z}}_{+} .
$$

Now, the result is a direct consequence of Proposition 5.3 and (v), (viii), (ix), and (x) of Lemma 5.2.

Next, we specialize the result of Proposition 5.4 to the case where there is no energy dissipation from each subsystem $\mathscr{G}_{i}$ of $G_{\text {; }}$ that is, $\sigma_{i i}=0, i=1, \ldots, q$. Note that in this case, $\mathrm{e}^{\mathrm{T}}\left(A-I_{q}\right)=0$, and hence $\operatorname{rank}\left(A-I_{q}\right) \leq q-1$. Furthermore, if $S=0$, it follows from (5.10) that $\mathbf{e}^{\mathrm{T}} \Delta E(k)=\mathbf{e}^{\mathrm{T}}\left(A-I_{q}\right) E(k)=0, k \in \overline{\mathbb{Z}}_{+}$, and hence the total energy of the isolated discrete-time large-scale nonlinear dynamical system $\mathscr{G}$ is conserved.

Proposition 5.5. Consider the discrete-time large-scale dynamical system $\mathscr{G}$ with energy balance equation given by (5.10). Assume that $\operatorname{rank}\left(A-I_{q}\right)=\operatorname{rank}\left(A^{2}-I_{q}\right)=q-1, \sigma_{i i}=$ $0, i=1, \ldots, q$, and $A=A^{\mathrm{T}}$. If $E_{0} \geq \geq 0$, and $S=0$, then the equilibrium state $\alpha \mathbf{e}, \alpha \geq 0$, of the isolated system $G$ is semistable and the steady-state energy distribution $E_{\infty}$ of the isolated discrete-time large-scale dynamical system $\mathscr{G}$ is given by

$$
E_{\infty}=\left[\frac{1}{q} \sum_{i=1}^{q} E_{i 0}\right] \mathbf{e} .
$$

If, in addition, for some $m \in\{1, \ldots, q\}, \sigma_{m m}>0$, then the zero solution $E(k) \equiv 0$ to (5.10) is globally asymptotically stable.

Proof. Note that since $\mathbf{e}^{\mathrm{T}}\left(A-I_{q}\right)=0$, it follows from (5.10) with $S(k) \equiv 0$ that $\mathbf{e}^{\mathrm{T}} \Delta E(k)=$ $0, k \geq 0$, and hence $\mathbf{e}^{\mathrm{T}} E(k)=\mathbf{e}^{\mathrm{T}} E_{0}, k \geq 0$. Furthermore, since by Proposition 5.3, the solution $E(k), k \geq k_{0}$, to (5.10) is nonnegative, it follows that $0 \leq E_{i}(k) \leq \mathbf{e}^{\mathrm{T}} E(k)=\mathbf{e}^{\mathrm{T}} E_{0}$, $k \geq 0, i=1, \ldots, q$. Hence, the solution $E(k), k \geq 0$, to (5.10) is bounded for all $E_{0} \in \overline{\mathbb{R}}_{+}^{q}$. Next, note that $\phi_{i j}(E)=\sigma_{i j}\left(E_{j}-E_{i}\right)$ and $\left(E_{i}-E_{j}\right) \phi_{i j}(E)=-\sigma_{i j}\left(E_{i}-E_{j}\right)^{2} \leq 0, E \in \overline{\mathbb{R}}_{+}^{q}$, $i \neq j, i, j=1, \ldots, q$, which implies that Axioms 1 and 2 are satisfied. Thus, $E=\alpha \mathbf{e}, \alpha \geq 0$, is the equilibrium state of the isolated large-scale dynamical system $\mathscr{G}$. Furthermore, define the Lyapunov function candidate $\mathscr{E}_{s}(E)=(1 / 2)(E-\alpha \mathbf{e})^{\mathrm{T}}(E-\alpha \mathbf{e}), E \in \overline{\mathbb{R}}_{+}^{q}$. Since $A$ is 
compartmental and symmetric, it follows from (ii) of Lemma 5.2 that

$$
\begin{aligned}
\Delta \mathscr{E}_{s}(E) & =\frac{1}{2}(A E-\alpha \mathbf{e})^{\mathrm{T}}(A E-\alpha \mathbf{e})-\frac{1}{2}(E-\alpha \mathbf{e})^{\mathrm{T}}(E-\alpha \mathbf{e}) \\
& =\frac{1}{2} E^{\mathrm{T}}\left(A^{2}-I_{q}\right) E \\
& \leq 0,
\end{aligned}
$$

which implies Lyapunov stability of the equilibrium state $\alpha \mathbf{e}, \alpha \geq 0$.

Next, consider the set $\mathscr{R} \triangleq\left\{E \in \overline{\mathbb{R}}_{+}^{q}: \Delta \mathscr{E}_{s}(E)=0\right\}=\left\{E \in \overline{\mathbb{R}}_{+}^{q}: E^{\mathrm{T}}\left(A^{2}-I_{q}\right) E=0\right\}$. Since $A$ is compartmental and symmetric, it follows from (ii) of Lemma 5.2 that $A^{2}-I_{q}$ is a negative semidefinite matrix, and hence $E^{\mathrm{T}}\left(A^{2}-I_{q}\right) E=0$ if and only if $\left(A^{2}-I_{q}\right) E=0$. Furthermore, since, by assumption, $\operatorname{rank}\left(A-I_{q}\right)=\operatorname{rank}\left(A^{2}-I_{q}\right)=q-1$, it follows that there exists one and only one linearly independent solution to $\left(A^{2}-I_{q}\right) E=0$ given by $E=\mathbf{e}$. Hence, $\mathscr{R}=\left\{E \in \overline{\mathbb{R}}_{+}^{q}: E=\alpha \mathbf{e}, \alpha \geq 0\right\}$. Since $\mathscr{R}$ consists of only equilibrium states of (5.10), it follows that $\mathcal{M}=\mathscr{R}$, where $\mathcal{M}$ is the largest invariant set contained in $\mathscr{R}$. Hence, for every $E_{0} \in \overline{\mathbb{R}}_{+}^{q}$, it follows from the LaSalle invariant set theorem that $E(k) \rightarrow \alpha \mathbf{e}$ as $k \rightarrow \infty$ for some $\alpha \geq 0$ and, hence, $\alpha \mathbf{e}, \alpha \geq 0$, is a semistable equilibrium state of (5.10). Furthermore, since the energy is conserved in the isolated large-scale dynamical system $\mathscr{G}$, it follows that $q \alpha=\mathrm{e}^{\mathrm{T}} E_{0}$. Thus, $\alpha=(1 / q) \sum_{i=1}^{q} E_{i 0}$, which implies (5.22).

Finally, to show that in the case where $\sigma_{m m}>0$ for some $m \in\{1, \ldots, q\}$, the zero solution $E(k) \equiv 0$ to (5.10) is globally asymptotically stable, consider the system ectropy $\mathscr{E}(E)=(1 / 2) E^{\mathrm{T}} E, E \in \overline{\mathbb{R}}_{+}^{q}$, as a candidate Lyapunov function. Note that Lyapunov stability of the zero equilibrium state follows from the previous analysis with $\alpha=0$. Next, note that

$$
\begin{aligned}
\Delta \mathscr{E}(E)= & \frac{1}{2} E^{\mathrm{T}}\left(A^{2}-I_{q}\right) E \\
= & \frac{1}{2} E^{\mathrm{T}}\left[(W-D)^{2}-I_{q}\right] E \\
= & \frac{1}{2} E^{\mathrm{T}}\left(W^{2}-I_{q}\right) E-\frac{1}{2} E^{\mathrm{T}}\left(W D+D W-D^{2}\right) E \\
= & \frac{1}{2} E^{\mathrm{T}}\left(W^{2}-I_{q}\right) E-\sum_{i=1, i \neq m}^{q} \sigma_{m m} \sigma_{m i} E_{m} E_{i} \\
& -\sigma_{m m}\left(W_{(m, m)}-\sigma_{m m}\right) E_{m}^{2}-\frac{1}{2} \sigma_{m m}^{2} E_{m}^{2}, \quad E \in \overline{\mathbb{R}}_{+}^{q} .
\end{aligned}
$$

Consider the set $\mathscr{R} \triangleq\left\{E \in \overline{\mathbb{R}}_{+}^{q}: \Delta \mathscr{E}(E)=0\right\}=\left\{E \in \overline{\mathbb{R}}_{+}^{q}: E_{1}=\cdots=E_{q}\right\} \cap\left\{E \in \overline{\mathbb{R}}_{+}^{q}: E_{m}=\right.$ $0, m \in\{1, \ldots, q\}\}=\{0\}$. Hence, the largest invariant set contained in $\mathscr{R}$ is given by $\mathcal{M}=\mathscr{R}=\{0\}$, and thus it follows from the LaSalle invariant set theorem that the zero solution $E(k) \equiv 0$ to (5.10) is globally asymptotically stable.

Finally, we examine the steady-state energy distribution for large-scale nonlinear dynamical systems $\mathscr{G}$ in case of strong coupling between subsystems; that is, $\sigma_{i j} \rightarrow \infty, i \neq j$. For this analysis, we assume that $A$ given by (5.10) is symmetric; that is, $\sigma_{i j}=\sigma_{j i}, i \neq j$, $i, j=1, \ldots, q$, and $\sigma_{i i}>0, i=1, \ldots, q$. Thus, $I_{q}-A$ is a nonsingular $M$-matrix for all values 
Wassim M. Haddad et al. 315

of $\sigma_{i j}, i \neq j, i, j=1, \ldots, q$. Moreover, in this case it follows that if $\sigma_{i j} / \sigma_{k l} \rightarrow 1$ as $\sigma_{i j} \rightarrow \infty$, $i \neq j$, and $\sigma_{k l} \rightarrow \infty, k \neq l$, then

$$
\lim _{\sigma_{i j} \rightarrow \infty, i \neq j}\left(I_{q}-A\right)^{-1}=\lim _{\sigma \rightarrow \infty}\left[D-\sigma\left(-q I_{q}+\mathbf{e e}^{\mathrm{T}}\right)\right]^{-1},
$$

where $D=\operatorname{diag}\left[\sigma_{11}, \ldots, \sigma_{q q}\right]>0$. The following lemmas are needed for the next result.

Lemma 5.6. Let $Y \in \mathbb{R}^{q \times q}$ be such that ind $(Y) \leq 1$. Then $\lim _{\sigma \rightarrow \infty}\left(I_{q}-\sigma Y\right)^{-1}=I_{q}-Y^{\#} Y$.

Proof. Note that

$$
\begin{aligned}
\left(I_{q}-\sigma Y\right)^{-1} & =I_{q}+\sigma\left(I_{q}-\sigma Y\right)^{-1} Y \\
& =I_{q}+\left(\frac{1}{\sigma} I_{q}-Y\right)^{-1} Y \\
& =I_{q}-\left(Y-\frac{1}{\sigma} I_{q}\right)^{-1} Y .
\end{aligned}
$$

Now, using the fact that if $A \in \mathbb{R}^{q \times q}$ and ind $A \leq 1$, then

$$
\lim _{\alpha \rightarrow 0}(A+\alpha I)^{-1} A=A A^{\#}=A^{\#} A,
$$

it follows that

$$
\lim _{\sigma \rightarrow \infty}\left(I_{q}-\sigma Y\right)^{-1}=I_{q}-\lim _{1 / \sigma \rightarrow 0}\left(Y-\frac{1}{\sigma} I_{q}\right)^{-1} Y=I_{q}-Y^{\#} Y
$$

which proves the result.

Lemma 5.7. Let $D \in \mathbb{R}^{q \times q}$ and $X \in \mathbb{R}^{q \times q}$ be such that $D>0$ and $X=-q I_{q}+\mathbf{e e}^{\mathrm{T}}$. Then,

$$
I_{q}-Y^{\#} Y=\frac{D^{1 / 2} \mathbf{e e}^{\mathrm{T}} D^{1 / 2}}{\mathbf{e}^{\mathrm{T}} D \mathbf{e}}
$$

where $Y \triangleq D^{-1 / 2} X D^{-1 / 2}$.

Proof. Note that

$$
Y=D^{-1 / 2}\left(-q I_{q}+\mathbf{e e}^{\mathrm{T}}\right) D^{-1 / 2}=-q D^{-1}+D^{-1 / 2} \mathbf{e e}^{\mathrm{T}} D^{-1 / 2} .
$$

Now, using the fact that if $N \in \mathbb{R}^{q \times q}$ is nonsingular and symmetric and $b \in \mathbb{R}^{q}$ is a nonzero vector, then

$$
\left(N+b b^{\mathrm{T}}\right)^{+}=\left(I-\frac{1}{b^{\mathrm{T}} N^{-2} b} N^{-1} b b^{\mathrm{T}} N^{-1}\right) N^{-1}\left(I-\frac{1}{b^{\mathrm{T}} N^{-2} b} N^{-1} b b^{\mathrm{T}} N^{-1}\right),
$$


it follows that

$$
-Y^{\#}=\frac{1}{q}\left(I_{q}-\frac{D^{1 / 2} \mathbf{e e}^{\mathrm{T}} D^{1 / 2}}{\mathbf{e}^{\mathrm{T}} D \mathbf{e}}\right) D\left(I_{q}-\frac{D^{1 / 2} \mathbf{e e}^{\mathrm{T}} D^{1 / 2}}{\mathbf{e}^{\mathrm{T}} D \mathbf{e}}\right) .
$$

Hence,

$$
\begin{aligned}
-Y^{\#} Y & =-\left(I_{q}-\frac{D^{1 / 2} \mathbf{e}^{\mathrm{T}} D^{1 / 2}}{\mathbf{e}^{\mathrm{T}} D \mathbf{e}}\right) D\left(I_{q}-\frac{D^{1 / 2} \mathbf{e e}^{\mathrm{T}} D^{1 / 2}}{\mathbf{e}^{\mathrm{T}} D \mathbf{e}}\right)\left(D^{-1}-\frac{1}{q} D^{-1 / 2} \mathbf{e}^{\mathrm{T}} D^{-1 / 2}\right) \\
& =-\left(I_{q}-\frac{D^{1 / 2} \mathbf{e}^{\mathrm{T}} D^{1 / 2}}{\mathbf{e}^{\mathrm{T}} D \mathbf{e}}\right) .
\end{aligned}
$$

Thus, $I_{q}-Y^{\#} Y=D^{1 / 2} \mathbf{e e}^{\mathrm{T}} D^{1 / 2} / \mathbf{e}^{\mathrm{T}} D \mathbf{e}$.

Proposition 5.8. Consider the discrete-time large-scale dynamical system $\mathscr{G}$ with energy balance equation given by (5.10). Let $S(k) \equiv S, S \in \mathbb{R}^{q \times q}, A \in \mathbb{R}^{q \times q}$ be compartmental and assume that $A$ is symmetric, $\sigma_{i i}>0, i=1, \ldots, q$, and $\sigma_{i j} / \sigma_{k l} \rightarrow 1$ as $\sigma_{i j} \rightarrow \infty, i \neq j$, and $\sigma_{k l} \rightarrow \infty, k \neq l$. Then the steady-state energy distribution $E_{\infty}$ of the discrete-time large-scale dynamical system $\mathscr{G}$ is given by

$$
E_{\infty}=\left[\frac{\mathbf{e}^{\mathrm{T}} S}{\sum_{i=1}^{q} \sigma_{i i}}\right] \mathbf{e} .
$$

Proof. Note that in the case where $\sigma_{i j} / \sigma_{k l} \rightarrow 1$ as $\sigma_{i j} \rightarrow \infty, i \neq j$, and $\sigma_{k l} \rightarrow \infty, k \neq l$, it follows that the corresponding limit of $\left(I_{q}-A\right)^{-1}$ can be equivalently taken as in (5.25). Next, with $D=\operatorname{diag}\left[\sigma_{11}, \ldots, \sigma_{q q}\right]$ and $X=-q I_{q}+\mathbf{e e}^{\mathrm{T}}$, it follows that $I_{q}-A=D-\sigma X=$ $D^{1 / 2}\left(I_{q}-\sigma D^{-1 / 2} X D^{-1 / 2}\right) D^{1 / 2}$. Now, it follows from Lemmas 5.6 and 5.7 that

$$
E_{\infty}=\lim _{\sigma_{i j} \rightarrow \infty, i \neq j}\left(I_{q}-A\right)^{-1} S=\frac{\mathbf{e e}^{\mathrm{T}}}{\mathbf{e}^{\mathrm{T}} D \mathbf{e}} S=\left[\frac{\mathbf{e}^{\mathrm{T}} S}{\sum_{i=1}^{q} \sigma_{i i}}\right] \mathbf{e},
$$

which proves the result.

Proposition 5.8 shows that in the limit of strong coupling, the steady-state energy distribution $E_{\infty}$ given by (5.20) becomes

$$
E_{\infty}=\lim _{\sigma_{i j} \rightarrow \infty, i \neq j}\left(I_{q}-A\right)^{-1} S=\left[\frac{\mathbf{e}^{\mathrm{T}} S}{\sum_{i=1}^{q} \sigma_{i i}}\right] \mathbf{e},
$$

which implies energy equipartition.

\section{Conclusion}

Motivated by energy flow modeling of large-scale interconnected systems, in this paper we develop discrete-time nonlinear compartmental models that are consistent with thermodynamic principles. Specifically, using a discrete-time large-scale systems perspective, we develop some of the key properties of thermodynamic systems involving conservation 
of energy and nonconservation of entropy and ectropy using dynamical systems theory. In addition, conditions were given under which steady-state energy and temperature distributions tend toward equipartition. Finally, the concept of entropy for a large-scale dynamical system is defined and shown to be consistent with the classical thermodynamic definition of entropy.

\section{Acknowledgments}

This research was supported in part by the Air Force Office of Scientific Research under Grant F49620-03-1-0178 and the National Science Foundation under Grant ECS0133038 .

\section{References}

[1] D. H. Anderson, Compartmental Modeling and Tracer Kinetics, Lecture Notes in Biomathematics, vol. 50, Springer, Berlin, 1983.

[2] A. Berman and R. J. Plemmons, Nonnegative Matrices in the Mathematical Sciences, Academic Press, New York, 1979.

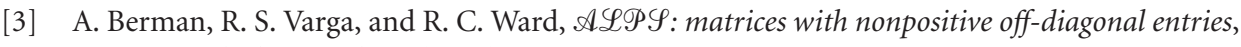
Linear Algebra Appl. 21 (1978), no. 3, 233-244.

[4] D. S. Bernstein and S. P. Bhat, Energy equipartition and the emergence of damping in lossless systems, Proc. IEEE Conf. Dec. Contr. (Las Vegas, Nev), 2002, pp. 2913-2918.

[5] D. S. Bernstein and D. C. Hyland, Compartmental modeling and second-moment analysis of state space systems, SIAM J. Matrix Anal. Appl. 14 (1993), no. 3, 880-901.

[6] R. W. Brockett and J. C. Willems, Stochastic control and the second law of thermodynamics, Proc. IEEE Conf. Dec. Contr. (San Diego, Calif), 1978, pp. 1007-1011.

[7] R. F. Brown, Compartmental system analysis: State of the art, IEEE Trans. Biomed. Engineering 27 (1980), 1-11.

[8] J. Brunet, Information theory and thermodynamics, Cybernetica 32 (1989), no. 1, 45-78.

[9] K. Godfrey, Compartmental Models and Their Application, Academic Press, London, 1983.

[10] E. P. Gyftopoulos and E. Çubukçu, Entropy: thermodynamic definition and quantum expression, Phys. Rev. E (3) 55 (1997), no. 4, 3851-3858.

[11] W. M. Haddad and V. Chellaboina, Stability and dissipativity theory for nonnegative dynamical systems: a unified analysis framework for biological and physiological systems, Nonlinear Anal. Real World Appl. 6 (2005), no. 1, 35-65.

[12] W. M. Haddad, V. Chellaboina, and E. August, Stability and dissipativity theory for discrete-time non-negative and compartmental dynamical systems, Internat. J. Control 76 (2003), no. 18, $1845-1861$.

[13] W. M. Haddad, V. Chellaboina, and S. G. Nersesov, Thermodynamics: A Dynamical Systems Approach, Princeton University Press, New Jersey, 2005.

[14] S. R. Hall, D. G. MacMartin, and D. S. Bernstein, Covariance averaging in the analysis of uncertain systems, Proc. IEEE Conf. Dec. Contr. (Tucson, Ariz), 1992, pp. 1842-1859.

[15] R. A. Horn and C. R. Johnson, Matrix Analysis, Cambridge University Press, Cambridge, 1985.

[16] J. A. Jacquez, Compartmental Analysis in Biology and Medicine, 2nd ed., University of Michigan Press, Michigan, 1985.

[17] J. A. Jacquez and C. P. Simon, Qualitative theory of compartmental systems, SIAM Rev. 35 (1993), no. 1, 43-79.

[18] R. H. Lyon, Statistical Energy Analysis of Dynamical Systems: Theory and Applications, MIT Press, Massachusetts, 1975. 
318 Thermodynamic modeling for discrete-time systems

[19] J. Meixner, On the foundation of thermodynamics of processes, A Critical Review of Thermodynamics (E. B. Stuart, B. Gal-Or, and A. J. Brainard, eds.), Mono Book Corp., Maryland, 1970, pp. 37-47.

[20] M. Pavon, Stochastic control and nonequilibrium thermodynamical systems, Appl. Math. Optim. 19 (1989), no. 2, 187-202.

[21] R. K. Pearson and T. L. Johnson, Energy equipartition and fluctuation-dissipation theorems for damped flexible structures, Quart. Appl. Math. 45 (1987), no. 2, 223-238.

[22] W. Sandberg, On the mathematical foundations of compartmental analysis in biology, medicine, and ecology, IEEE Trans. Circuits and Systems 25 (1978), no. 5, 273-279.

[23] J. C. Willems, Dissipative dynamical systems. I. General theory, Arch. Rational Mech. Anal. 45 (1972), 321-351.

[24] J. M. Ziman, Models of Disorder, Cambridge University Press, Cambridge, 1979.

Wassim M. Haddad: School of Aerospace Engineering, Georgia Institute of Technology, Atlanta, GA 30332-0150, USA

E-mail address:wm.haddad@aerospace.gatech.edu

Qing Hui: School of Aerospace Engineering, Georgia Institute of Technology, Atlanta, GA 303320150, USA

E-mail address: qing_hui@ae.gatech.edu

Sergey G. Nersesov: School of Aerospace Engineering, Georgia Institute of Technology, Atlanta, GA 30332-0150, USA

E-mail address: sergey_nersesov@ae.gatech.edu

Vijaysekhar Chellaboina: Department of Mechanical, Aerospace and Biomedical Engineering, The University of Tennessee, Knoxville, TN 37996-2210, USA

E-mail address: chellaboina@utk.edu 